Pacific Journal of Mathematics

ESSENTIAL CENTRAL SPECTRUM AND RANGE FOR
ELEMENTS OF A YON NEUMANN ALGEBRA 


\section{ESSENTIAL CENTRAL SPECTRUM AND RANGE FOR ELEMENTS OF A VON NEUMANN ALGEBRA}

\section{HERBERT HALPERN}

A closed two-sided ideal $\mathscr{F}$ in a von Neumann algebra $\mathscr{A}$ is defined to be a central ideal if $\sum A_{i} P_{i}$ is in $\mathscr{F}$ for every set $\left\{P_{i}\right\}$ of orthogonal projections in the center $\mathscr{Z}$ of $\mathscr{A}$ and every bounded subset $\left\{A_{i}\right\}$ of $\mathscr{F}$. Central ideals are characterized in terms of the existence of continuous fields and their form is completely determined.

If $\mathscr{F}$ is a central ideal of $\mathscr{A}$ and $A \in \mathscr{A}$, then $A_{0} \in \mathscr{Z}$ is said to be in the essential central spectrum of $A$ if $A_{0}-A$ is not invertible in $\mathscr{A}$ modulo the smallest closed ideal containing $\mathscr{F}$ and $\zeta$ for every maximal ideal $\zeta$ of $\mathscr{\gamma}$. It is shown that the essential central spectrum is a nonvoid, strongly closed subset of $\mathscr{F}$ and that it satisfies many of the relations of the essential spectrum of operators on Hilbert space. Let $\mathscr{A} \sim$ be the space of all bounded $\mathscr{Z}$-module homomorphisms of $\mathscr{A}$ into $\mathscr{\%}$. The essential central numerical range of $A \epsilon$ $\mathscr{A}$ with respect to $\mathscr{F}$ is defined to be $\mathscr{K}_{\mathscr{S}}(A)=\{\phi(A) \mid \phi \epsilon$ $\left.\mathscr{A} \sim,\|\phi\| \leqq 1, \phi(1)=P_{\mathscr{S}}, \phi(\mathscr{\mathcal { F }})=(0)\right\}$. Here $P_{\mathscr{F}}$ is the orthogonal complement of the largest central projection in $\mathscr{F}$. The essential central numerical range is shown to be a weakly closed, bounded, $\mathscr{\digamma}$-convex subset of $\mathscr{Z}$. It possesses many of the properties of the essential numerical range but in a form more suited to the fact that $A$ is in $\mathscr{A}$ rather than a bounded operator. It is shown that if $\mathscr{A}$ is properly infinite and $\mathscr{F}$ is the ideal of finite elements (resp. the strong radical) of $\mathscr{A}$, then $\mathscr{K}_{\mathcal{Y}}(A)$ is the intersection of $\mathscr{\digamma}$ with the weak (resp. uniform) closure of the convex hull of $\left\{U A U^{-1} \mid U\right.$ unitary in $\mathscr{A}\}$.

In a final section, we give some applications of these facts. We extend a result of J. G. Stampfli [19] to show that the range of a derivation on a von Neumann algebra is never uniformly dense. We also prove a theorem on self-adjoint commutators using a calculation of M. David [5].

2. Central ideals. Let $\mathscr{A}$ be a von Neumann algebra with center $\mathscr{Z}$. For any subset $\mathscr{B}$ of $\mathscr{A}$ let $(\mathscr{B})$ denote the set of all projections of $\mathscr{B}$. Throughout this paper all ideals will be assumed to be closed two-sided ideals. An ideal $\mathscr{F}$ in $\mathscr{A}$ is said to be a central ideal or a $\not{Z}$-ideal if given a norm bounded set $\left\{A_{i} \mid i \in I\right\}$ of elements of $\mathscr{J}$ and a corresponding set $\left\{P_{i} \mid i \in I\right\}$ of mutually orthogonal projections in $\mathscr{z}$, then the sum $\sum A_{i} P_{i}$, which exists in the strong topology, is also in $\mathscr{F}$. (Similar definitions were used by I. Kaplansky 
$[22, \S 1]$ and M. Goldman $[13 ; \S 4]$ in the theory of $A W^{*}$-modules; however, here there is no canonical inner product.) Any ideal $\mathscr{J}$ in $\mathscr{A}$ is contained in a smallest central ideal $\langle\mathscr{I}\rangle$ given by $\langle\mathscr{F}\rangle=$ $\left\{\sum\left\{A_{i} P_{i} \mid i \in I\right\} \mid\left\{A_{i} \mid i \in I\right\}\right.$ is a bounded subset of $\mathscr{J}$ and $\left\{P_{i} \mid i \in I\right\}$ is a mutually orthogonal subset of (F) of sum 1\} ([19], remarks preceding corollary to (a5) implies (a1)). If $\mathscr{J}$ a central ideal in $\mathscr{A}$ and if $A$ is an element of $\mathscr{A}$, then it is clear that there is an element $P$ in

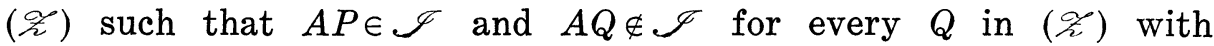
$0<Q \leqq 1-P$. The following definition is now possible.

Definition 2.1. Let $\mathscr{A}$ be a von Neumann algebra and let $\mathscr{J}$ be a central ideal of $\mathscr{A}$. Then $P_{\mathscr{S}}$ will denote the orthogonal comple-

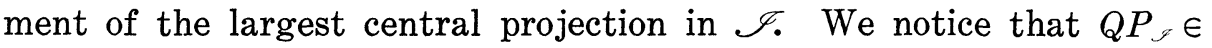
$\mathscr{F}$ for a central projection $Q$ implies $Q P_{\mathscr{S}}=0$.

We now describe central ideals with regard to finite element

Proposition 2.2. Let $\mathscr{A}$ be a semi-finite von Neumann algebra with center $\mathscr{L}$, let $\mathscr{F}$ be a central ideal of $\mathscr{A}$, and let $\mathscr{A} P$ be the weak closure of $\mathscr{F}$, where $P \in(\mathscr{L})$. Then $\mathscr{F}$ contains every finite projection of A majorized by $P$.

Proof. Let $F$ be a finite projection of $\mathscr{A}$ majorized by $P$. Let $Q$ be an element of ( $\mathscr{z})$ such that $F Q \in \mathscr{J}$ and $F R \notin \mathscr{J}$ for every $R$ in ( $\not 2$ ) with $0<R \leqq 1-Q$ (preliminary remarks). We note that $Q^{\prime}=1-Q \leqq P$. We obtain a contradiction by assuming that $Q^{\prime} \neq 0$. Since the weak closure of $\mathscr{J}$ is $\mathscr{A} P$ and since linear combinations of projections are dense in $\mathscr{F}$, there is a projection $E$ in $\mathscr{J}$ with $E Q^{\prime} \neq 0$. There is an $R$ in $\left(\mathscr{F} Q^{\prime}\right)$ such that $E R<F R$ and $F\left(Q^{\prime}-R\right) \prec E\left(Q^{\prime}-R\right)$. Either $E R \neq 0$ or $E\left(Q^{\prime}-R\right) \neq 0$. Now if $E R \neq 0$, there is nonzero $S$ in $(\not{z} R)$ and projections $E_{1}, \cdots, E_{n}$ in $\mathscr{A}$ such that $E S=E_{1} \sim E_{2} \sim \cdots \sim E_{n}$ and $F S-\sum E_{i} \prec E_{1}$. This means that $F S$ is in $\mathscr{F}$. This is contrary to the choice of $Q$, so we must assume that $E\left(Q^{\prime}-R\right) \neq 0$. But this also implies that $F\left(Q^{\prime}-R\right)$ is in $\mathscr{F}$. So we must conclude that $Q^{\prime}=0$. Hence, we have shown that every finite projection majorized by $P$ is in $\mathscr{J}$.

Corollary 2.3. An ideal in a finite von Neumann algebra is a central ideal if and only if it is weakly closed.

Proof. If the ideal $\mathscr{J}$ in the finite von Neumann algebra $\mathscr{A}$ is weakly closed, then there is a central projection $P$ in $\mathscr{A}$ such that $\mathscr{J}=\mathscr{A P}[$ [9, I, 3, Theorem 2, Corollary 2]. Obviously the ideal 
$\mathscr{A} P$ is a central ideal of $\mathscr{A}$.

Conversely, let $\mathscr{J}$ be a central ideal of $\mathscr{A}$. Let $P$ be the central projection of $\mathscr{A}$ such that the weak closure of $\mathscr{J}$ is $\mathscr{A} P$. Then $\mathscr{F}$ contains every finite projection majorized by $P$; in particular, it contains $P$ itself. So $\mathscr{J}=\mathscr{A} P$ and $\mathscr{F}$ is weakly closed.

We now describe central ideals for an arbitrary von Neumann algebra $\mathscr{A}$ with center $\mathscr{Z}$. Let $P$ be a projection in $\mathscr{Z}$ and let $E$ be a properly infinite projection in $\mathscr{A}$ majorized by $P$. (By convention we assume that 0 is a properly infinite projection in a finite algebra $\mathscr{2}$.) Let $\left(\mathscr{J}_{P}(E)\right)$ be the set of all projections in $\mathscr{A}$ given by $\left(\mathscr{I}_{p}(E)\right)=\{F \in(\mathscr{A}) \mid F \leqq P$ and $Q E \prec Q F$ for some $Q \in(\mathscr{\not})$ implies $Q E=0\}$. Let $\mathscr{I}_{P}(E)$ be the ideal generated by $\left(\mathscr{J}_{P}(E)\right)$.

We shall use the following lemma of F. B. Wright [32; §2].

Lemma. Suppose $\mathscr{P}$ is a set of projections on a von Neumann algebra A that satisfies the following properties:

(1) if $E \in(\mathscr{A}), F \in \mathscr{P}$ and $E \prec F$, then $E \in \mathscr{P}$; and

(2) if $E$ and $F$ are in $\mathscr{P}$, then the least upper bound lub $\{E, F\}$ of $E$ and $F$ is in $\mathscr{P}$.

Then the set of projections of the ideal generated by $\mathscr{P}$ is exactly $\mathscr{P}$.

THEOREM 2.4. Let $\mathscr{A}$ be a von Neumann algebra with center $\mathscr{Z}$. In order for the ideal $\mathscr{I}$ in $\mathscr{A}$ to be a central ideal, it is a necessary and sufficient condition that there exist a projection $P$ in $\mathscr{Z}$ and a properly infinite projection $E$ majorized by $P$ with $\mathscr{F}=\mathscr{F}_{P}(E)$.

REMARK. The sufficiency is an adaptation of the proof we gave for a special case in an earlier paper [18, Proposition 2.1].

Proof. Let $E$ be a properly infinite projection majorized by the central projection $P$. We show that $\mathscr{J}_{P}(E)=\mathscr{J}$ is a central ideal. Let $P_{1}$ and $P_{2}$ be orthogonal central projections of sum 1 such that $\mathscr{A} P_{1}$ is a finite algebra and $\mathscr{A} P_{2}$ is a properly infinite. It is sufficient to show that $\mathscr{J} P_{i}$ is a central ideal in $\mathscr{A} P_{i}(i=1,2)$. However, we have that $\mathscr{J} P_{i}$ is generated by $\left(\mathscr{F} P_{i}\right)=\left\{F \in\left(A P_{i}\right) \mid F \leqq P P_{i}\right.$, $E Q P_{i} \prec F Q$ for some $Q$ in $\left(\not 2 P_{i}\right)$ implies $\left.E P_{i} Q=0\right\}$. Now setting $E_{i}=E P_{i}$, we obtain a properly infinite projection in $\mathscr{A} P_{i}$ so that $\mathscr{J} P_{i}=\mathscr{J}_{P P_{i}}\left(E_{i}\right)$. Hence, there is no loss of generality in assuming that $\mathscr{A}$ is either finite or properly infinite.

Let $\mathscr{A}$ be finite. Then $E=0$ and $\left(\mathscr{I}_{P}(0)\right)=\{F \in(A) \mid F \leqq P\}$. Hence $\mathscr{F}=\mathscr{A} P$ and so $\mathscr{F}$ is a central ideal.

Now assume that $\mathscr{A}$ is properly infinite. There is no loss of 
generality in the assumption that $P=1$. We show that $\mathscr{F}$ satisfies properties (1) and (2) of the lemma of F. B. Wright. By the definition of $(\mathscr{J})$ is clear that it satisfies property (1). Now let $E_{1}$ and $E_{2}$ be in $(\mathscr{J})$. Since lub $\left\{E_{1}, E_{2}\right\}-E_{1} \prec E_{2}$ [21, Theorem 5.4], we have that lub $\left\{E_{1}, E_{2}\right\}-E_{1}$ is in $(\mathscr{J})$ by (1). So there is no loss of generality in the assumption that $E_{1}$ and $E_{2}$ are orthogonal. There is $Q \in(\mathscr{C})$ such that $Q E_{1} \prec Q E_{2}$ and $(1-Q) E_{2} \prec(1-Q) E_{1}$. Since $Q\left(E_{1}+E_{2}\right) \in(\mathscr{F})$ and $(1-Q)\left(E_{1}+E_{2}\right) \in(\mathscr{F})$ implies that $E_{1}+E_{2} \in$ $(\mathscr{J})$, there is no loss of generality in the assumption that $E_{1} \prec E_{2}$. There is a $Q \in(\mathscr{Z})$ such that $Q E_{2}$ is finite and $(1-Q) E_{2}$ is properly infinite. Hence, we may assume that either $E_{2}$ is finite or properly infinite. If $E_{2}$ is finite, then $E_{1}$ is finite since $E_{1} \prec E_{2}$ and so $E_{1}+E_{2}$ is finite. [9, III, 2]. If $Q$ is a central projection with $Q E<Q\left(E_{1}+E_{2}\right)$, then $Q E$ is finite and so $Q E=0$. So we are left with the situation that $E_{1} \prec E_{2}, E_{1} E_{2}=0$, and $E_{2}$ is properly infinite. Because $E_{2}$ is properly infinite, there are projections $F_{1}, F_{2}$ satisfying the relations: $F_{1} \sim F_{2} \sim E_{2}, F_{1} F_{2}=0$, and $F_{1}+F_{2}=E_{2}$. [9; III, 8, Corollary 2]. We have that $E_{1}+E_{2} \sim E_{1}+F_{2}<F_{2}+F_{2}=E_{2}$. By property (1) of the lemma, we conclude that $E_{1}+E_{2} \in(\mathscr{I})$. Hence $(\mathscr{J})$ satisfies properties (1) and (2) of the lemma and this means that the set of projections of the ideal $\mathscr{J}$ generated by $(\mathscr{J})$ is precisely $(\mathscr{J})$. Now we show $\mathscr{J}$ is a central ideal. Let $\left\{A_{i} \mid i \in I\right\}$ be a bounded set in $\mathscr{F}$ and let $\left\{Q_{i} \mid i \in I\right\}$ be an orthogonal subset of ( $\mathscr{\Sigma}$ ) of sum 1. For every $\varepsilon>0$ and every $i \in I$ there is a projection $F_{i}$ in $(\mathscr{F})$ such that $\left\|A_{i}-A_{i} F_{i}\right\| \leqq \varepsilon$. Then $\sum F_{i} Q_{i}=F$ is in $(\mathscr{F})$. Indeed, if $E Q \prec F Q$ for some $Q$ in ( $\mathscr{z})$, then $E\left(Q_{i} Q\right) \prec F\left(Q_{i} Q\right)=F_{i}\left(Q_{i} Q\right)$ for every $i \in I$. Thus $(E Q) Q_{i}=0$ for every $i \in I$ and $E Q=\sum(E Q) Q_{i}=0$. This means that $F \in(\mathscr{F})$. However, we have that

$$
\left\|\sum A_{i} Q_{i}-\left(\sum A_{i} Q_{i}\right) F\right\| \leqq \operatorname{lub}\left\|A_{i}-A_{i} F_{i}\right\| \leqq \varepsilon .
$$

Since $\left(\sum A_{i} Q_{i}\right) F$ is in $\mathscr{J}$ and since $\mathscr{F}$ is uniformly closed, we have that $\sum A_{i} Q_{i} \in \mathscr{F}$. This proves that $\mathscr{J}$ is a central ideal.

We now show that every central ideal $\mathscr{J}$ is of the form $\mathscr{J}_{P}(E)$. Given a nonzero $P \in(\mathscr{Z})$ it is sufficient to prove that there is a pro-

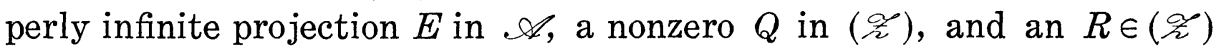
with $R \leqq Q \leqq P$ such that $\mathscr{J}_{R}(E R) Q=\mathscr{J} Q$. Indeed, suppose we have verified this statement. Let $\left\{P_{i} \mid i \in I\right\}$ be a maximal set of mutually orthogonal nonzero central projections such that for each $P_{i}$ there is a properly infinite projection $E_{i}$, and a $Q_{i} \in(\not{z})$ majorized by $P_{i}$ such that $\mathscr{J}_{Q_{i}}\left(E Q_{i}\right) P_{i}=\mathscr{J} P_{i}$. By the maximality of $\left\{P_{i}\right\}$, we conclude that $\sum P_{i}=1$. Setting $E=\sum E_{i} Q_{i}$ (resp. $Q=\sum Q_{i}$ ) we obtain a properly infinite (resp. central) projection $E$ majorized by $Q$ such that $\mathscr{I}_{Q}(E)=\mathscr{F}$. In fact, since $\mathscr{I}_{Q}(E)$ and $\mathscr{J}$ are generated by 
their respective projections, it is sufficient to show that $\left(\mathscr{I}_{Q}(E)\right)=(\mathscr{I})$, But we may verify immediately that $\left(\mathscr{F}_{Q}(E)\right) P_{i}=\left(\mathscr{J}_{Q_{i}}\left(E_{i} Q_{i}\right)\right)$, and so we have that $F \in\left(\mathscr{J}_{Q}(E)\right)$ if and only if $F P_{i} \in\left(\mathscr{F}_{Q_{i}}\left(E_{i} Q_{i}\right)\right)=\left(\mathscr{F} P_{i}\right)$ for every $P_{i}$ since $\mathscr{I}_{Q}(E)$ is a central ideal by the first part of this theorem. However, the ideal $\mathscr{F}$ is also a central ideal and thus $F \in\left(\mathscr{F}_{Q}(E)\right.$ ) if and only if $F \in(\mathscr{F})$. So it is sufficient to verify the required statement. We do this in the next paragraph.

Let $P$ be a nonzero element in $(\mathscr{L})$. Since we are looking for a nonzero central projection $Q$ majorized by $P$, we may assume at the outset that $P=1$ and that either $\mathscr{A}$ is finite or $\mathscr{A}$ is properly infinite. If $\mathscr{A}$ is finite there is a $Q$ in $(\mathscr{K})$ with $\mathscr{J}=\mathscr{A} Q$ (corollary 2.3). Then we verify immediately that $\mathscr{J}=\mathscr{J}_{Q}(0)$. Hence, we may assume that $\mathscr{A}$ is properly infinite. Suppose that there is a projection $P \neq 1$ in $(\mathscr{K})$ such that $A P=A$ for every $A$ in $\mathscr{F}$. Then we have that $\mathscr{F}_{0}(0)(1-P)=0=\mathscr{F}(1-P)$. So we may assume that $\mathscr{F}$ is weakly dense in $\mathscr{A}$. Now suppose that $P, \neq 1$. Then the nonzero central projection $Q=1-P_{\mathscr{S}}$ is in $\mathscr{F}$. This means $\mathscr{J} Q=$ $\mathscr{A} Q=\mathscr{F}_{Q}(0)$. Hence, we may pass to the case that $P_{S}=1$. By making a further reduction if necessary, we may assume that 1 is the sum of an infinite set $\left\{E_{i} \mid i \in I\right\}$ of orthogonal, equivalent, $\sigma$-finite projections [9, III, 1, Lemma 1]. Let $\mathscr{S}(I)$ be the family of all subsets $s$ of $I$ such that there is a nonzero projection $P_{s}$ in $\mathscr{F}$ with

$$
\sum\left\{E_{i} \mid i \in s\right\} Q \notin \mathscr{J}
$$

for every nonzero $Q \in\left(\mathscr{F} P_{s}\right)$. The family $\mathscr{S}(I)$ is nonvoid since $I \in$ $\mathscr{S}(I)$ with $P_{I}=1$. There is an $s_{0} \in \mathscr{S}(I)$ such that Card $s_{0} \leqq$ Card $s$ for every $s \in \mathscr{S}(I)$. We may assume that $P_{s_{0}}=1$. Let $\sum\left\{E_{i} \mid i \in s_{0}\right\}=$ $E$; we notice that $E$ is a properly infinite projection of central support 1. We show that $\mathscr{J}_{1}(E)=\mathscr{F}(E)$ is equal to $\mathscr{F}$. First we prove that $(\mathscr{S}) \subset(\mathscr{I}(E))$. Let $F \in(\mathscr{S})$. If $E P \prec F P$ for some $P \in(\mathscr{F})$, then by choice of $s_{0}$ we have that $E P=0$. So $F \in(\mathscr{F}(E))$ by definition and hence $(\mathscr{J}) \subset(\mathscr{F}(E))$. To show the converse relation $(\mathscr{J}(E)) \subset(\mathscr{S})$ we consider two cases: (i) Card $s_{0}$ is finite, and (ii) Card $s_{0}$ is infinite. For case (i) we have that $E$ is a $\sigma$-finite projection of central support 1 . Then we have that $(\mathscr{J}(E)$ ) is exactly the set of finite projections of . [9; III, 8, Corollary 5]. But by our preliminary reduction $\mathscr{S}$ is weakly dense in $\mathscr{A}$ and therefore contains all finite projections of $\mathscr{C}$ (Proposition 2.2). So $(\mathscr{J}(E)) \subset(\mathscr{F})$. Now we consider case (ii). Let $F \in(\mathscr{J}(E))$. Since $\mathscr{F}$ is a central ideal, there is a $P \in(\mathscr{F})$ such that $P F \in \mathscr{F}$ and $Q F \notin \mathscr{J}$ for every nonzero $Q$ in $(\mathscr{Z}(1-P))$. We obtain a contradiction by assuming $1-P \neq 0$. Because $\mathscr{F}$ contains all finite projections (Proposition 2.2), we have that $F(1-P)$ is properly infinite 
with central support $1-P$. We may find a nonzero projection $Q$ in $(\mathscr{Z}(1-P))$ such that $F Q$ is the sum of a set $\left\{F_{i} \mid i \in s\right\}$ of orthogonal, equivalent, properly infinite $\sigma$-finite projections [9; III, 1, Lemma 7]. We have that $F_{1} \sim E_{j} Q$ for every $i \in s$ and $j \in s_{0}$. [9; III, 8, Corollary 5]. Since $\sum\left\{F_{i} \mid i \in s\right\}=F Q \prec E Q=\sum\left\{E_{i} Q \mid i \in s_{0}\right\}$, and since Card $s_{0}$ is infinite, we have that Card $s \leqq$ Card $s_{0}$ [9; III, 1, Lemma 6]. If Card $s_{0} \leqq$ Card $s$, we would have a contradiction in that $E Q \prec F Q$ and $E Q \neq 0$. Thus Card $s \neq \operatorname{Card} s_{0}$. But if $s^{\prime}$ is a subset of $\mathscr{S}(I)$ with Card $s^{\prime}=$ Card $s$, then $\sum\left\{E_{i} \mid i \in s^{\prime}\right\} Q^{\prime} \sim F Q^{\prime}$ for every $Q^{\prime}$ in $(\mathscr{L} Q)$ and so $\sum\left\{E_{i} \mid i \in s^{\prime}\right\} Q^{\prime} \notin \mathscr{J}$ for every nonzero $Q^{\prime}$ in $(\mathscr{L} Q)$. This contradicts the choice of $s_{0}$. Hence, $1-P=0$ and $F \in(\mathscr{F})$. So in case (ii) we have $(\mathscr{J}(E)) \subset(\mathscr{J})$. Therefore, we have completed the crucial step, and so there is an $E \in(\mathscr{A})$ and a $Q \in(\mathscr{L})$ with $\mathscr{J}=\mathscr{I}_{Q}(E)$.

Now let $E$ be a properly infinite projection majorized by the central projection $P$ in the von Neumann algebra $\mathscr{A}$. Let $Q$ be the central projection of $\mathscr{A}$ such that $\mathscr{A} Q$ is equal to the weak closure of

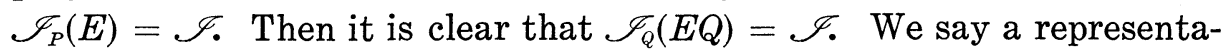
tion $\mathscr{J}_{P}(E)$ for a central ideal $\mathscr{J}$ is in canonical form if $\mathscr{A} P$ is the weak closure of $\mathscr{J}_{P}(E)$.

Proposition 2.5. Let $\mathscr{J}_{P}(E)$ and $\mathscr{J}_{Q}(F)$ be two central ideals of a von Neumann algebra. $\mathscr{A}$ that are represented in canonical form. Then $\mathscr{I}_{P}(E)=\mathscr{J}_{Q}(F)$ if and only if $P=Q$ and $E \sim F$.

Proof. If $P=Q$ and $E \sim F$, then it is clear that $\mathscr{I}_{P}(E)=\mathscr{F}_{Q}(F)$.

Now let $\mathscr{J}_{P}(E)=\mathscr{I}_{Q}(F)=\mathscr{F}$. Since $\mathscr{A} P=$ weak closure $\mathscr{J}=$ $\mathscr{A} Q$, we have that $P=Q$. Now let $R$ be the largest central projection majorized by $P$ such that $R E \sim R F$. Suppose $R^{\prime}=P-R \neq 0$. There is a central projection $R^{\prime \prime}$ majorized by $R^{\prime}$ such that $R^{\prime \prime} E \prec$ $R^{\prime \prime} F$ and $\left(R^{\prime}-R^{\prime \prime}\right) F \prec\left(R^{\prime}-R^{\prime \prime}\right) E$. If $R^{\prime \prime} \neq 0$, then $S R^{\prime \prime} F \prec S R^{\prime \prime} E$ for some central projection $S$ implies that $S R^{\prime \prime} F=0$. Otherwise, we would have that $S R^{\prime \prime} E \sim S R^{\prime \prime} F$ and so $R$ would not be the largest central projection with $R E \sim R F$. This means that $R^{\prime \prime} E \in \mathscr{F}$. Hence $R^{\prime \prime} E=0$ and so $\mathscr{J} R^{\prime \prime}=\mathscr{A} R^{\prime \prime}$. This means that $F R^{\prime \prime}=0$ and consequently that $E R^{\prime \prime} \sim F R^{\prime \prime}$. This is a contradiction. A similar contradiction arises if $R^{\prime}-R^{\prime \prime} \neq 0$. So we must have that $R=P$, i.e., $E \sim F$.

REMARK 2.6. In the sequel we assume all representations of central ideals are in canonical form.

CoROLlaRY 2.7. Let $\mathscr{A}$ be a von Neumann algebra and let $\mathscr{J}$ be a central ideal of $\mathscr{A}$ given by $\mathscr{J}=\mathscr{I}_{P}(E)$ in canonical form. 
Then in order that $P_{,}=1$, a necessary and sufficient condition is that $P$ be the central support of $E$.

Proof. If the central support of $E$ is $Q$, then from the definition of $\mathscr{S}_{P}(E)=\mathscr{S}$, it is clear that $P-Q \in \mathscr{S}$. This means that $P_{\mathscr{V}} \neq 1$ if $P-Q \neq 0$. Conversely, if $1-P_{\mathcal{S}} \neq 0$, then $\left(1-P_{\mathcal{S}}\right) E=0$. But $\left(1-P_{\mathcal{I}}\right) \leqq P$ and thus $E$ cannot have central support $P$.

Let $\mathscr{A}$ be a von Neumann algebra with center $\mathscr{Z}$. Let $Z$ be the spectrum of $\mathscr{x}$. Let $C_{C}(Z)$ be the set of order-continuous functions of $Z$ into the set of cardinal numbers. J. Tomiyama [30] showed that there is a dimension function $D$ of $\mathscr{C}$ into $C_{C}(Z)$ such that $D(E) \leqq D(F)$ if and only if $E \prec F$. W. Wils [31] described the range of $D$ in $C_{C}(Z)$ as being a certain subset $\Delta$ of functions in $C_{C}(Z)$. Although it is not important in the sequel, one may see that the set of projections of a central ideal $\mathscr{F}_{P}(E)$ satisfies a certain dimension relation relative to $P$ and $E$. We therefore feel justified in introducing a name for the following relation.

DEFINITION 2.8. Let $\mathscr{J}$ be a central ideal in a von Neumann algebra $\mathscr{A}$. Let $P$ be a central projection and let $E$ be a properly infinite projection majorized by $P$ with $\mathscr{F}=\mathscr{F}_{P}(E)$. A projection $F$ in $\mathscr{A}$ is said to have dimension greater than that of $\mathscr{F}$ if $F$ has central support $P_{\mathscr{F}}$ and if $F>E P_{\mathscr{S}}$ (in symbols, $\operatorname{dim} F>\operatorname{dim} \mathscr{J}$ ).

The following proposition characterizes the projections whose dimension is greater than the dimension of $\mathscr{F}$.

Proposition 2.9. Let $\mathscr{A}$ be a von Neumann algebra and let $\mathscr{F}$ be a central ideal of $\mathscr{M}$. Then a projection $F$ of $\mathscr{A}$ has dimension greater than that of $\mathscr{S}$ if and only if $F$ has central support $P_{\mathscr{Y}}$ and $F Q \in \mathscr{J}$ for some central projection $Q$ implies $F Q=0$.

Proof. Let $\mathscr{Z}$ be the center of $\mathscr{F}$. Let $E \in(\mathscr{A})$ and let $P \in(\mathscr{Z})$ so that $\mathscr{F}_{P}(E)$ represents $\mathscr{F}$ in canonical form. First let $F \in(\mathscr{A})$ with central support $P_{\mathscr{Y}}$ such that $Q F \in \mathscr{F}$ for some $Q \in(\mathscr{F})$ implies $Q F=0$. There is an $R \in(\mathscr{Y})$ such that $R E \prec R F$ and such that $R^{\prime} E \prec R^{\prime} F$ for $R^{\prime} \in(Z(1-R))$ implies $R^{\prime}=0$. Then $F P(1-R) \in$ $\mathscr{J}_{P}(E)$ by definition and so $F P(1-R)=0$. Thus we obtain that $F P R=F P$. So $E P_{\mathscr{G}}=E P P_{\mathscr{S}} \prec F P \leqq F$, i.e. $\operatorname{dim} F>\operatorname{dim} \mathscr{\mathscr { T }}$.

Conversely, let $\operatorname{dim} F>\operatorname{dim} \mathscr{S}$. Then by definition we have that $F$ has central support $P_{\mathscr{S}}$. Let $Q \in(\mathscr{F})$ and let $Q F \in \mathscr{F}$. We have that $E P, \prec F$ implies that $E Q P_{\mathscr{S}} \in \mathscr{S}$ (lemma of F. B. Wright). Since $E Q P_{\mathscr{Y}} \prec E Q P_{\mathscr{Y}}$, we have that $E Q P_{\mathscr{F}}=0$ and thus $Q P P_{\mathscr{C}} \in \mathscr{I}^{\text {. }}$ 
By definition of $P_{\mathscr{S}}$ we find that $Q P P_{\mathscr{S}}=0$. Also $\mathscr{J}(1-P)=(0)$ and so $Q F=Q P F+Q(1-P) F=0$.

Now we can give some examples.

EXAMPLE 2.10. In a factor algebra, every ideal is a central ideal.

EXAMPLE 2.11. In a semi-finite algebra $\mathscr{A}$, the ideal $\mathscr{J}$ generated by all finite projections of $\mathscr{A}$ is a central ideal. If $\mathscr{A}$ is finite, then $\mathscr{J}=\mathscr{A}$; if $\mathscr{A}$ is properly infinite, then $\mathscr{J}=\mathscr{J}_{1}(E)$, where $E$ is a properly infinite projection of central support 1 for which there is a set $\left\{P_{i}\right\}$ of mutually orthogonal central projections of sum 1 such that $E P_{i}$ is $\sigma$-finite for every $P_{i}$ [8; III, 1, Lemma 7].

EXAMPLE 2.12. If $\mathscr{A}$ is a properly infinite von Neumann algebra, then the strong radical $\mathscr{J}$ (i.e. the intersection of all maximal ideals) is a central ideal with $\mathscr{J}=\mathscr{J}_{1}(1)$.

3. The essential central spectrum. Let $\mathscr{A}$ be a von Neumann algebra with center $\mathscr{Z}$. If $\mathscr{J}$ is an ideal in $\mathscr{A}$, let $\mathscr{A}(\mathscr{J})$ denote the algebra $\mathscr{A}$ reduced modulo $\mathscr{F}$, and let $A(\mathscr{J})$ denote the image of an element $A$ under the canonical homomorphism of $\mathscr{A}$ into $\mathscr{A}(\mathscr{I})$. The algebra $\mathscr{A}(\mathscr{J})$ is a $C^{*}$-algebra under the norm $\|A(\mathscr{I})\|=$ glb $\{\|A+B\| \mid B \in \mathscr{J}\}$. If $\zeta$ is an element in the spectrum $Z$ of $\mathscr{\gamma}$, let

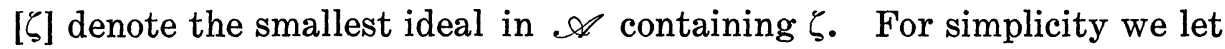
$\mathscr{A}([\zeta])$ and $A([\zeta])$ be denoted by the symbols $\mathscr{A}(\zeta)$ and $A(\zeta)$, respectively. Then J. Glimm [12; Lemma 10] has shown that for fixed $A \in$ $\mathscr{A}$ the $\operatorname{map} \zeta \rightarrow\|A(\zeta)\|$ is continuous on the spectrum $Z$. For every $A$ in $\mathscr{A}$ and $\zeta$ in $Z$, the norm $\|A(\zeta)\|$ is equal to $\|A(\zeta)\|=\operatorname{glb}\{\|A P\| P \in$ $(\mathscr{\Sigma})$ and $P^{\wedge}(\zeta)=1$. . Here $P^{\wedge}$ denotes the Gelfand transform of $P$. If $\mathscr{F}_{1}$ and $\mathscr{I}_{2}$ are ideals in $\mathscr{A}$, then the algebraic sum $\mathscr{I}_{1}+\mathscr{F}_{2}$ is also an ideal of $\mathscr{A}$. In the sequel we denote the sum $\mathscr{J}+[\zeta]$ of an ideal $\mathscr{J}$ and the special ideal [ $\zeta$ ] formed from $\zeta \in Z$ by $\mathscr{J}(\zeta)$. For an element $A$ in $\mathscr{A}$, we denote the spectrum of $A(\mathscr{I}(\zeta))$ in $\mathscr{A}(\mathscr{I}(\zeta))$ by $\operatorname{Sp} A(\mathscr{I}(\zeta))$.

The next lemma is used repeatedly.

Lemma 3.1. Let $\mathscr{A}$ be a von Neumann algebra, let $\mathscr{Z}$ be the center of $\mathscr{A}$, let $P \in(\mathscr{Z})$, let $Z$ be the spectrum of $\mathscr{Z}$, and let $\mathscr{J}$ be a central ideal of $\mathscr{A}$. If $A$ is an element of $\mathscr{A}$ such that $f_{A}(\zeta)=$ $\|A(\mathscr{J}(\zeta))\|$ vanishes for every $\zeta$ in the support of $P$ given by supp $P=$ $\left\{\zeta \in Z \mid P^{\wedge}(\zeta)=1\right\}$, then the element $A P$ is in $\mathscr{J}$.

Proof. For every $\zeta$ in supp $P$ and $\varepsilon>0$ there is a $B_{\zeta}$ in $\mathscr{J}$ such that $\left\|\left(A-B_{\zeta}\right)(\zeta)\right\|<\varepsilon$. Hence there is a $P_{\zeta}$ in $\left(\varkappa_{\check{\Sigma}}\right)$ with $P_{\zeta}^{\wedge}(\zeta)=1$ 
such that $\left\|\left(A-B_{\zeta}\right) P_{\zeta}\right\|<\varepsilon$. Using the fact that supp $P$ is compact, we may find a set $P_{1}, \cdots, P_{n}$ of orthogonal projections in $\mathscr{F}$ of sum $P$ and a corresponding set $B_{1}, \cdots, B_{n}$ in $\mathscr{F}$ such that

$$
\left\|A P-\sum B_{i} P_{i}\right\|=\operatorname{lub}\left\|\left(A-B_{i}\right) P_{i}\right\|<\varepsilon .
$$

Since $\mathscr{F}$ is closed, the element $A P$ is in $\mathscr{F}$.

We characterize those ideals $\mathscr{F}$ for which $\zeta \rightarrow\|A(\mathscr{F}(\zeta))\|$ is continuous on $Z$ for every $A$ in $\mathscr{A}$.

THEOREM 3.2. Let $\mathscr{A}$ be a von Neumann algebra, let \& be the center of $\mathscr{A}$, and let $Z$ be the spectrum of $\mathscr{\not}$. Let $\mathscr{F}$ be an ideal of $\mathscr{A}$. In order that $f_{A}(\zeta)=\|A(\mathscr{J}(\zeta))\|$ be a continuous function on $Z$ for every $A$ in $\mathscr{A}$, a necessary and sufficient condition is that $\mathscr{F}$ be a central ideal of $\mathscr{A}$.

Proof. The sufficiency follows by a proof that is virtually the same as the one we gave in the corollary of (a5) implies (a1) of [19].

Conversely, let $f_{A}$ be continuous on $Z$ for every $A$ in $\mathscr{A}$. We show that $\mathscr{A}$ is a central ideal. If $\left\{A_{i} \mid i \in I\right\}$ is a bounded subset

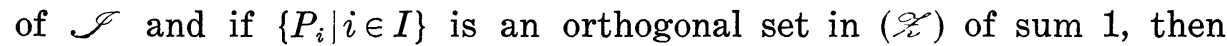
we prove that $A=\sum A_{i} P_{i}$ is in $\mathscr{A}$. Indeed, the set $\cup_{i}\left\{\zeta \in Z \mid P_{i}^{\wedge}(\zeta)=1\right\}$ is a dense set of $Z$ on which $f_{A}(\zeta)$ vanishes since $f_{A}(\zeta)=\left\|A_{i}(\mathscr{F}(\zeta))\right\|=$ 0 whenever $P_{i}^{\wedge}(\zeta)=1$. By the continuity of $f_{A}$, we see that $f_{A}$ vanishes on $Z$. Hence, the element $A$ is in $\mathscr{J}$ by Lemma 3.1.

REMARK 3.3. If $\mathscr{J}$ is the strong radical of a properly infinite von Neumann algebra, then $\mathscr{J}(\zeta)=\mathscr{J}+[\zeta]$ is the unique maximal ideal which contains $\zeta$ [24 and 15, Proposition 2.3].

Now we prove the main result of this section. It is convenient to separate the following lemma.

LEMmA 3.4. Let $\mathscr{2}$ be a commutative von Neumann algebra and let $X_{1}, \cdots, X_{n}$ be closed sets which cover the spectrum $Z$ of $\mathscr{Z}$. Then there are orthogonal projections $R_{1}, \cdots, R_{n}$ in (关) of sum 1 such that $\left\{\zeta \in Z \mid R_{i}^{\wedge}(\zeta)=1\right\} \subset X_{i}$ for $1 \leqq i \leqq n$.

Proof. Let $\left\{P_{i} \mid i \in I\right\}$ be a maximal set of nonzero mutually orthogonal projections such that for each $i \in I$ there is an $i(j)$ with $1 \leqq i(j) \leqq n$ so that $Y_{i}=\left\{\zeta \in Z \mid P_{i}^{\wedge}(\zeta)=1\right\} \subset X_{i(j)}$. We obtain a contradiction if $P=1-\sum P_{i} \neq 0$. Indeed, the set $Y=\left\{\zeta \in Z \mid P^{\wedge}(\zeta)=1\right\}$ is nonvoid and is covered by the closed sets $Y \cap X_{1}, \cdots, Y \cap X_{n}$. By the Baire category theorem one of the set $Y \cap X_{m}$ has a nonvoid 
interior in $Y$. This means that there is nonzero projection $Q$ in $\mathscr{Z}$ such that $\left\{\zeta \in Z \mid Q^{\wedge}(\zeta)=1\right\} \subset Y \cap X_{m}$. This contradicts the maximality of $\left\{P_{i}\right\}$. We must have that $\sum P_{i}=1$. The remainder of the proof consists in adding the projections $P_{i}$. Let $I_{j}=\left\{i \in I \mid Y_{i} \subset X_{j}\right\}$ for $1 \leqq j \leqq n$ and let $R_{j}=\sum\left\{P_{i} \mid i \in I_{j}-\cup\left\{I_{k} \mid 0 \leqq k \leqq j-1\right\}\right\}$ for $1 \leqq j \leqq n$. Here $I_{0}=\varnothing$. Then it is clear that $R_{1}, R_{2}, \cdots, R_{n}$ satisfy the requirements of the lemma.

THEOREM 3.5. Let $\mathscr{A}$ be a von Neumann algebra with center $\mathscr{Z}$, let $\mathscr{F}$ be a central ideal of $\mathscr{A}$, and let $A$ be an element of $\mathscr{A}$. Let $X_{0}$ be a closed subset of the complex plane $C$ such that the intersection $S(\zeta)$ of $X_{0}$ with the spectrum (resp. left-spectrum, right-spectrum, the intersection of the left-spectrum and the right-spectrum) of $A(\mathscr{F}(\zeta)$ ) is nonvoid for every $\zeta$ in the spectrum $Z$ of $\mathscr{Z}$. Then there is an element $A_{0}$ in the center of $\mathscr{A}$ such that $A_{0}^{\wedge}(\zeta) \in S(\zeta)$ for every $\zeta$ in $Z$.

Proof. We first prove that there exists $A_{0}$ in $\mathscr{Z}$ such that $A_{0}^{\wedge}(\zeta)$ is in the intersection $S(\zeta)$ of $X_{0}$ with the spectrum $\operatorname{Sp} A(\mathscr{I}(\zeta))$ of $A(\mathscr{J}(\zeta))$ for every $\zeta$ in $Z$. Since $\operatorname{Sp} A(\mathscr{F}(\zeta))$ is contained in $\operatorname{Sp} A$, there is no loss of generality in assuming $X_{0} \subset \mathrm{Sp}(A)$. We prove the theorem by an approximation argument that involves decomposing the space $Z$.

For every compact set $X$ in the complex plane, let $X(Z)=\{\zeta \in$ $Z \mid X \cap S(\zeta) \neq \varnothing\}$. We show that $X(Z)$ is closed in $C$. Let $\left\{\zeta_{i}\right\}$ be a net in $X(Z)$ converging to $\zeta$. Let $\alpha_{i} \in S\left(\zeta_{i}\right) \cap X$; by passing to a subnet, we may assume that $\left\{\alpha_{i}\right\}$ converges to $\alpha \in X \cap X_{0}$. Arguing by contradiction we show that $\alpha \in \operatorname{Sp} A(\mathscr{I}(\zeta))$. If $\alpha \notin \operatorname{Sp} A(\mathscr{J}(\zeta))$, then there is a $B \in \mathscr{A}$ with

$$
\begin{aligned}
& \|(B(\alpha-A)-1)(\mathscr{F}(\zeta))\|= \\
& \|((\alpha-A) B-1)(\mathscr{J}(\alpha))\|=0 .
\end{aligned}
$$

By Theorem 3.2, we see that there is a $\zeta_{i}$ and $\alpha_{i}$ such that

$$
\left\|\left(B\left(\alpha_{i}-A\right)-1\right)\left(\mathscr{J}\left(\zeta_{i}\right)\right)\right\|<1
$$

and $\left\|\left(\left(\alpha_{i}-A\right) B-1\right)\left(\mathscr{J}\left(\zeta_{i}\right)\right)\right\|<1$. This means that $\alpha_{i} \notin \operatorname{Sp} A\left(\mathscr{I}\left(\zeta_{i}\right)\right)$ and this is contrary to assumption. So $\alpha \in X(Z)$ and $X(Z)$ is closed.

We now begin the approximation argument by decomposing $Z$ into subsets on which we shall approximate $A_{0}$. Suppose we have, for every $m$ less than or equal to the natural number $n$, constructed sets of integers $I_{m}=\left\{1,2, \cdots, p_{m}\right\}$ such that for every $s$ in $I_{1} \times \cdots \times$ $I_{m}=I(m)$ there is a compact subset $X(s)$ of $C$ of diameter $\leqq 2^{-m}$ and a $P(s)$ in $(\mathscr{L})$ which satisfies the following properties:

(1) For $s \in I(m), \cup\left\{X(s ; j) \mid j \in I_{m+1}\right\}=X(s)$ whenever $1 \leqq m<n$ and 


$$
\cup\{X(j) \mid j \in I(1)\}=X_{0}
$$

(2) Supp $P(s)=\left\{\zeta \in Z \mid P(s)^{\wedge}(\zeta)=1\right\} \subset X(s)(Z)$ for every $s \in I(m)(1 \leqq$ $m \leqq n)$; and

(3) for $s \in I(m),\left\{P(s ; j) \mid j \in I_{m+1}\right\}$ is a set of orthogonal projections of sum $P(s)$ whenever $1<m<n$ and $\{P(j) \mid j \in I(1)\}$ is a set of orthogonal projections of sum 1 .

We shall construct a set $I_{n+1}=\left\{1, \cdots, p_{n+1}\right\}$, compact sets $X(s)(s \in$ $\left.I(n+1)=I_{1} \times \cdots \times I_{n+1}\right)$ of diameter $\leqq 2^{-(n+1)}$ in the complex plane, and projections $P(s)(s \in I(n+1))$ in $\mathscr{z}$ which satisfy (1), (2), (3). Indeed, let $\left\{Y_{j} \mid j \in I_{n+1}\right\}$ be compact sets of diameter $\leqq 2^{-(n+1)}$ which cover $X_{0}$. Let $X(s, j)=X(s) \cap Y_{j}$ for $s \in I(n)$ and $j \in I_{n+1}$. Then $\{X(s) \mid s \in I(m), m=1,2, \cdots, n+1\}$ satisfies property (1). Now let $s$ be fixed in $I(n)$; we have that $U\left\{X(s ; j)(Z) \mid j \in I_{n+1}\right\}=X(s)(Z)$. Since supp $P(s)$ is contained in $X(s)(Z)$, the sets $X(s ; j)(Z) \cap \operatorname{supp} P(s)\left(j \in I_{n+1}\right)$ form a closed cover of supp $P(s)$. By the Lemma 3.4, there are orthogonal central projections $P(s ; j)\left(j \in I_{n+1}\right)$ of sum $P(s)$ such that

$$
\operatorname{supp} P(s ; j) \subset X(s ; j)(Z)
$$

for every $j \in I_{n+1}$. Thus $P(s)(s \in I(n+1))$ satisfies (2) and (3).

We continue by induction to construct $I(n)$, compact sets $X(s)$ $(s \in I(n))$ of diameter $\leqq 2^{-n}$, and central projections $P(s)(s \in I(n))$ satisfying (1), (2), and (3) for every $n=1,2, \cdots$. We notice that if $X(s)$ is void then $P(s)=0$.

We now construct the approximating elements. Let $n=1,2, \cdots$ be fixed. If $s \in I(n)$, let $\alpha(s) \in X(s)$ if $X(s)$ is non-void, and $\alpha(s)=0$ if $X(s)$ is void. Let $A_{n}=\sum\{\alpha(s) P(s) \mid s \in I(n)\}$. Then $A_{n}$ is an element in the center of $\mathscr{A}$.

We show that $\left\{A_{n}\right\}$ is a Cauchy sequence. Indeed, we have that

$$
\left\|A_{n}-A_{n+1}\right\|=\operatorname{lub}\left\{\left\|\left(A_{n}-A_{n+1}\right) P(s)\right\| \mid s \in I(n+1)\right\}
$$

since $\sum\{P(s) \mid s \in I(n+1)\}=\sum\{P(s) \mid s \in I(n)\}=\cdots=1$. However, if $s \in I(n+1)$ is of the form $s=\left(s^{\prime} ; j\right)$ with $s^{\prime} \in I(n)$ and $j \in I_{n+1}$, then

$$
\left\|\left(A_{n}-A_{n+1}\right) P(s)\right\|=\left\|\left(\alpha\left(s^{\prime}\right)-\alpha(s)\right) P(s)\right\| \leqq 2^{-n}
$$

since $\alpha(s) \in X\left(s^{\prime}\right)$ whenever $P(s) \neq 0$. Hence, we obtain that

$$
\left\|A_{n}-A_{n+1}\right\| \leqq 2^{-n}
$$

for every $n=1,2, \cdots$ and so $\left\{A_{n}\right\}$ is a Cauchy sequence in $\mathscr{z}$.

We show that the limit $A_{0}$ of $\left\{A_{n}\right\}$ satisfies the requirements of the Theorem 3.5. Let $\zeta$ be an arbitrary point in $Z$. Given $\varepsilon>0$ we show that there is $\alpha \in S(\zeta)$ such that $\left|A_{0}^{\wedge}(\zeta)-\alpha\right| \leqq \varepsilon$. Since $S(\zeta)$ is 
closed and since $\varepsilon>0$ is arbitrary, this will mean that $A_{0}^{\wedge}(\zeta) \in S(\zeta)$. Let $m$ be a natural number with $2^{-m+2}<\varepsilon$. Then $\left|A_{0}^{\wedge}(\zeta)-A_{m}^{\wedge}(\zeta)\right| \leqq$ $\left\|A_{0}-A_{m}\right\|<2^{-1} \varepsilon$. There is an $s \in I(m)$ such that $P(s)^{\wedge}(\zeta)=1$ since $\sum\{P(s) \mid s \in I(m)\}=1$. By property (2), we have that $\zeta$ is in $X(s)(Z)$. So there is an element $\alpha$ in $X(s)$ such that $\alpha \in S(\zeta)$. However we have that $A_{m}^{\wedge}(\zeta)=\alpha(s) \in X(s)$, and so $|\alpha(s)-\alpha|<2^{-m}$ since the diameter of $X(s)$ is less than $2^{-m}$. Now we obtain that $\left|A_{0}^{\wedge}(\zeta)-\alpha\right| \leqq \varepsilon$, and by the preceding remarks that $A_{0}^{\wedge}(\zeta) \in S(\zeta)$. This completes the proof for the case of $X_{0} \cap \operatorname{Sp} A(\mathscr{F}(\zeta)) \neq \varnothing$.

We may prove the existence of an element $A_{0}$ in $\mathscr{C}$ such that $\left(A_{0}-A\right)(\mathscr{J}(\zeta))$ is not left (resp. right, left nor right) invertible in $\mathscr{A}(\mathscr{I}(\zeta))$ and $A_{0}^{\wedge}(\zeta) \in X_{0}$ by the same proof we just gave for an invertible element by using the additional fact that, for any element $B$ in a Banach algebra $\mathscr{B}$ with identity, the set of all complex $\alpha$ such that $\alpha-B$ is not left (resp. right, left nor right) invertible is a non-void compact set $([26 ; 1.5 .4$ and 1.4.6]; also cf. [11; Theorem 3.1]).

The following definition is now meaningful.

Definition 3.6. Let $\mathscr{A}$ be a von Neumann algebra, let $\mathscr{Z}$ be the center of $\mathscr{A}$ and let $Z$ be the spectrum of $\mathscr{Z}$. Then the essential central spectrum $\mathscr{Z}-\mathrm{Sp}_{\mathcal{S}} A$ of an element $A$ in $\mathscr{A}$ with respect to the central ideal $\mathscr{J}$ is the set of all $A_{0}$ in $\mathscr{Z}$ such that $A_{0}^{\wedge}(\zeta) \in$ $\operatorname{Sp} A(\mathscr{F}(\zeta))$ for every $\zeta \in Z$. The left-essential (resp. right-essential) central spectral $\mathscr{Z}-\mathrm{Sp}_{\mathcal{S}}^{e} A$ (resp. $\mathscr{Z}-\mathrm{Sp}_{\mathcal{F}}^{r} A$ ) of $A$ with respect to $\mathscr{J}$ is defined in a similar manner. The intersection $\mathscr{Z}-\mathrm{Sp}_{\mathscr{C}}^{b} A=$ $\left(\mathscr{L}-\mathrm{Sp}_{\digamma}^{e} A\right) \cap\left(\mathscr{Z}-\mathrm{Sp}_{\digamma}^{r} A\right)$ is called the two-sided essential central spectrum of $A$ with respect to $\mathscr{F}$.

Remark 3.7. All sets defined in Definition 3.6 are non-void (Theorem 3.5).

REMARK 3.8. For every $A_{0} \in \mathscr{C}-\mathrm{Sp}, A$, we have that $A_{0}(1-$

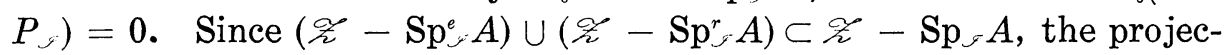
tion $1-P_{\mathscr{S}}$ annihilates the other essential central spectrums.

We note that these definitions correspond to the usual ones if $\mathscr{A}$ is the algebra of all bounded operators on a Hilbert space and $\mathscr{F}$ is the ideal of compact operators.

Proposition 3.9. Let $\mathscr{A}$ be a von Neumann algebra. Then the essential (resp. left-, right-essential) central spectrum of an element $A$ in $\mathscr{A}$ with respect to a central ideal $\mathscr{F}$ is closed in the strong operator topology. 
Proof. Let $\left\{A_{i}\right\}$ be a net in the essential central spectrum of $A$ with respect to $\mathscr{F}$ which converges strongly to $A_{0}$ in the center $\mathscr{Z}$ of $\mathscr{A}$. There is a net $\left\{P_{n}\right\}$ of mutually orthogonal central projections of sum 1 such that for each $P_{n}$ there is a sequence $\left\{A_{i(n)}\right\}$ in $\cup_{i}\left\{A_{i}\right\}$ with $\lim A_{i(n)} P_{n}=A_{0} P_{n}$ (uniformly) [28; Corollary 13.1]. Since $A_{i(n)}^{\wedge}(\zeta) \in$ $\operatorname{Sp} A(\mathscr{F}(\zeta))$ for every $\zeta$ in the spectrum $Z$ of $\mathscr{Z}$ and since $\operatorname{Sp} A(\mathscr{J}(\zeta))$ is closed, we have that $A_{0}^{\wedge}(\zeta) \in \operatorname{Sp} A(\mathscr{I}(\zeta))$ for every $\zeta$ in the dense subset $X=\cup_{n}\left\{\zeta \in Z \mid P_{n}^{\wedge}(\zeta)=1\right\}$ of $Z$ [7]. Let $\left\{\zeta_{i}\right\}$ be a net in $X$ which converges to $\zeta$ in $Z$. If $A_{0}^{\wedge}(\zeta) \notin \operatorname{Sp} A(\mathscr{J}(\zeta))$, then there is a $B$ in $\mathscr{A}$ with

$$
\left\|\left(B\left(A_{0}-A\right)-1\right)(\mathscr{F}(\zeta))\right\|=\left\|\left(\left(A_{0}-A\right) B-1\right)(\mathscr{F}(\zeta))\right\|=0 .
$$

This means that there is a $\zeta_{i}$ with

$$
\left.\left\|\left(B\left(A_{0}-A\right)-1\right)\left(\mathscr{F}\left(\zeta_{i}\right)\right)\right\|<1 \text { and } \|\left(A_{0}-A\right) B-1\right)\left(\mathscr{J}\left(\zeta_{i}\right)\right) \|<1
$$

and thus that $A_{0}^{\wedge}\left(\zeta_{i}\right)$ is not in $\operatorname{Sp} A\left(\mathscr{J}\left(\zeta_{i}\right)\right)$. Hence, we must have that $A_{0}^{\wedge}(\zeta)$ is in $\operatorname{Sp} A(\mathscr{J}(\zeta))$ for every $\zeta$ in the closure $Z$ of $X$. This proves that $\mathscr{Z}-\mathrm{Sp}_{A} A$ is strongly closed.

The statements concerning the left- and right-essential central spectra are proved in an analogous fashion.

For future reference we note some simple facts in the following proposition.

Proposition 3.10. Let $\mathscr{A}$ be a von Neumann algebra with center $\mathscr{2}$ on the Hilbert space $H$, let $\mathscr{F}$ be a central ideal in $\mathscr{A}$, let $P_{1}$ and $P_{2}$ be orthogonal projections of sum 1 in $\mathscr{\Sigma}$, and let $A$ be an element of $\mathscr{A}$. Let $\mathscr{A}_{i}$ be the von Neumann algebra $\mathscr{A} P_{i}$ with center $\mathscr{Z}_{i}=\mathscr{Z} P_{i}$ on the Hilbert space $P_{i} H$, let $\mathscr{I}_{i}$ be the central ideal $\mathscr{F} P_{i}$ in $\mathscr{A}_{i}$, and let $A_{i}$ be the element $A P_{i}$ in $\mathscr{A}_{i}$ for $i=1,2$. Then $\mathscr{Z}-\operatorname{Sp}_{\mathscr{J}} A=\left\{B_{1}+B_{2} \mid B_{i} \in \mathscr{Z}_{i}-\operatorname{Sp}_{i} A_{i}, i=1,2\right\}$.

REMARK. A similar statement holds for the left- and rightessential central spectrums.

Proof. This follows from the fact that the spectrum of $\mathscr{Z}_{i}$ is $\left\{\zeta P_{i} \mid \zeta \in Z, P_{i}^{\wedge}(\zeta)=1\right\}$, where $Z$ is the spectrum of $\mathscr{Z}$, and thus that

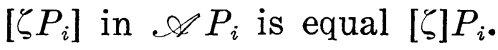

We now restrict our attention to self-adjoint elements. We note that the essential central spectrum of a self-adjoint element consists of self-adjoint elements.

Proposition 3.11. Let $\mathscr{A}$ be a von Neumann algebra, let $\mathscr{2}$ be 
the center of $\mathscr{A}$, and let $A$ be a self-adjoint element of $\mathscr{A}$. Then there are elements $C_{u}$ and $C_{l}$ in the essential central spectrum $\mathscr{F}$ $\operatorname{Sp}_{\mathcal{S}} A$ of $A$ with respect to the central ideal $\mathscr{F}$ such that $C_{l} \leqq C \leqq C_{u}$ for every $C$ in $\not$ \& $-\operatorname{Sp}_{\mathscr{S}} A$.

Proof. The set $\mathscr{C}-\mathrm{Sp}_{\mathcal{S}} A$ is a monotonely increasing net in $\mathscr{Z}$. Indeed, if $C$ and $C^{\prime}$ are in $\mathscr{Z}-\mathrm{Sp}_{J} A$, then there is a $P \in(\mathscr{Z})$ such that lub $\left\{C, C^{\prime}\right\}=P C+(1-P) C^{\prime}$. Since $P C+(1-P) C^{\prime}$ is in $\mathscr{Z}-\mathrm{Sp}_{\sigma} A$ (by 3.10), the set $\mathscr{Z}-\mathrm{Sp}_{\mathscr{S}} A$ is monotonely increasing. Then the least upper bound $C_{u}$ of $\mathscr{Z}-\mathrm{Sp}_{\mathscr{C}} A$ is the strong limit of elements in $\mathscr{Z}-\mathrm{Sp}_{S} A$ and so $C_{u}$ is in the essential central spectrum of $A$ with respect to $\mathscr{F}$ (Proposition 3.8).

In an analogous manner, we may show that $\mathscr{z}-\mathrm{Sp}_{,} A$ is monotonely decreasing and thus we may find a greatest lower bound $C_{l}$ for $\mathscr{Z}-\operatorname{Sp}_{\mathscr{J}} A$ in $\mathscr{Z}-\operatorname{Sp}_{\mathscr{T}} A$.

Proposition 3.12. Let $\mathscr{A}$ be a von Neumann algebra with center $\mathscr{Z}$, let $\mathscr{I}$ be a central ideal of $\mathscr{A}$, and let $A$ be a self-adjoint element of $\mathscr{A}$. Let $C_{u}$ and $C_{l}$ be the least upper bound and the greatest lower bound of the essential central spectrum of $A$ with respect to $\mathscr{F}$, respectively. Then $C_{u}^{\wedge}(\zeta)=\operatorname{lub} \operatorname{Sp} A(\mathscr{J}(\zeta))$ and $C_{l}^{\wedge}(\zeta)=\operatorname{glb} \operatorname{Sp} A(\mathscr{I}(\zeta))$ for every $\zeta$ in the spectrum $Z$ of $\not$.

Proof. Since $C_{u}^{\wedge}(\zeta) \in \operatorname{Sp} A(\mathscr{F}(\zeta))$ for every $\zeta \in Z$, we have that $C_{u}^{\wedge}(\zeta) \leqq \alpha_{\zeta}=\operatorname{lub} \operatorname{Sp} A(\mathscr{I}(\zeta))$, for every $\zeta \in Z$. Conversely, we obtain a contradiction if we assume that $\alpha_{\zeta}-C_{u}^{\wedge}(\zeta)=2 \varepsilon>0$ for some $\zeta \in Z$. Indeed, let $E$ be the spectral projection of $A-C_{u}$ corresponding to the interval $[\varepsilon,+\infty)$. Because $\left(A-C_{u}\right)(1-E) \leqq \varepsilon(1-E)$, we have that $E(\mathscr{I}(\zeta)) \neq 0$. Hence, there is a $P \in(\mathscr{L})$ such that $P^{\wedge}(\zeta)=1$ and $E\left(\mathscr{\mathcal { F }}\left(\zeta^{\prime}\right)\right) \neq 0$ for all $\zeta^{\prime}$ in $\operatorname{supp} P=\left\{\zeta^{\prime} \in Z \mid P^{\wedge}\left(\zeta^{\prime}\right)=1\right\}$ (Theorem 3.2). Since $\varepsilon E \leqq\left(A-C_{u}\right) E$, we have that $\operatorname{Sp}\left(A-C_{u}\right)\left(\mathscr{J}\left(\zeta^{\prime}\right)\right) \cap[\varepsilon,+\infty) \neq$ $\varnothing$ for all $\zeta^{\prime} \in \operatorname{supp} P$. Reducing to the algebra $\mathscr{A} P$ with center $\mathscr{L} P$, we see that $S\left(\zeta^{\prime}\right)=\operatorname{Sp}\left(A-C_{u}\right) P\left((\mathscr{\mathcal { S } P})\left(\zeta^{\prime}\right)\right) \cap[\varepsilon,+\infty)$ is non-void for every $\zeta^{\prime}$ in the spectrum $X$ of $\mathscr{L} P$. Because $\mathscr{F} P$ is a central ideal in $\mathscr{A} P$, we may find a $B$ in $\mathscr{\digamma}$ such that $(B P)^{\wedge}\left(\zeta^{\prime}\right) \in S\left(\zeta^{\prime}\right)$ for every $\zeta^{\prime} \in X$ (Theorem 3.5). If $D$ is an arbitrary element in $\not \check{\gamma}_{-}-\operatorname{Sp}_{\mathcal{J}}\left(A-C_{u}\right)$, then $P B+(1-P) D=B^{\prime}$ is in $\mathscr{Z}-\operatorname{Sp}_{\mathcal{I}}\left(A-C_{u}\right)$ (Proposition 3.10), and consequently, the element $B^{\prime \prime}=B^{\prime}+C_{u}$ is in $\not 2-\mathrm{Sp}_{3} A$. But we have that $B^{\prime \prime} P+C_{u}(1-P)$ is in $\mathscr{\digamma}-\operatorname{Sp}_{\mathscr{S}} A$ (Proposition 3.10) and that $B^{\prime \prime} P+C_{u}(1-P) \geqq C_{u}+\varepsilon P$. This contradicts the definition of $C_{u}$. Thus we must have that $C_{u}^{\wedge}(\zeta)=\operatorname{lubSp} A(\mathscr{J}(\zeta))$ for every $\zeta \in Z$.

A similar proof holds for $C_{l}$. 
The following proposition shows that if $A_{0}$ is in the essential central spectrum of $A$ with respect to $\mathscr{F}$, then $A_{0}-A$ is small on a large subspace with respect to $\mathscr{F}$.

Proposition 3.13. Let $\mathscr{A}$ be a von Neumann algebra, let $\mathscr{F}$ be a central ideal of $\mathscr{A}$, let $A$ be a self-adjoint element of $\mathscr{A}$, let $A_{0}$ be an element of the essential central spectrum of $A$ with respect to $\mathscr{I}$, and let $\varepsilon>0$. If $F$ is the spectral projection of $A_{0}-A$ corresponding to the interval $[-\varepsilon, \varepsilon]$, then $F P \in \mathscr{J}$ for some central projection $P$ implies $P \in \mathscr{J}$ (i.e. $P \leqq 1-P_{\mathcal{S}}$ ).

Proof. Let $P$ be a central projection with $P F \in \mathscr{F}$. We show $P \in \mathscr{F}$. We may assume that $P \neq 0$. Let $\zeta$ be a point in the spectrum of the center of $\mathscr{A}$ such that $P^{\wedge}(\zeta)=1$. We have that

$$
\left(A_{0}-A\right)(\mathscr{\rho}(\zeta))=\left(A_{0}-A\right)(1-F)(\mathscr{I}(\zeta)) \text {. }
$$

If $(1-F)(\mathscr{J}(\zeta)) \neq 0$, then $\left(A_{0}-A\right)(\mathscr{J}(\zeta))$ is invertible in $\mathscr{A}(\mathscr{J}(\zeta))$. Since this is not possible, we have that $1(\mathscr{J}(\zeta))=0$. This means that $P \in \mathscr{J}(\zeta)$. Since $\zeta$ with the property $P^{\wedge}(\zeta)=1$ is arbitrary in the last relation, we have that $P \in \mathscr{J}$ by Lemma 3.1.

We now characterize the essential central spectrum of a selfadjoint element in terms of the canonical form of a central ideal (cf. Remark 2.6ff. and Definition 2.8).

Proposition 3.14. Let. $\mathscr{A}$ be a von Neumann algebra with no finite type I direct summand, let $\mathscr{I}$ be a central ideal of $\mathscr{A}$, and let $A$ be a self-adjoint element in $\mathscr{A}$. An element $A_{0}$ is in the essential central spectrum of $A$ with respect to $\mathscr{J}$ if and only if there is an orthogonal sequence $\left\{E_{n}\right\}$ of projections in $\mathscr{A}$ of dimension greater than $\operatorname{dim} \mathscr{J}$ such that $A E_{n}(\mathscr{J})=E_{n} A(\mathscr{F})$ and $\left\|\left(A_{0}-A\right) E_{n}(\mathscr{J})\right\| \leqq n^{-1}$ for every $n=1,2, \cdots$ and $A_{0}=A_{0} P_{\mathcal{S}}$.

Proof. Let $A_{0}$ be in the essential central spectrum of $A$ with respect to $\mathscr{F}$. There is no loss of generality in the assumption that $P_{S}=1$ and that $A_{0}=0$. [9; III, 5, Problem 7]. Let $F_{n}$ be the spectral projection of $A$ corresponding to the interval $\left[-n^{-1}, n^{-1}\right]$ for $n=1,2, \cdots$; then we have that $\left\{F_{n}\right\}$ is a monotonely decreasing sequence of projections such that $\operatorname{dim} F_{n}>\operatorname{dim} \mathscr{F}$ (Propositions 3.13 and 2.9).

Let $\mathscr{J}$ be represented in the form $\mathscr{F}=\mathscr{J}_{S}(E)$ (2.4-2.6). Now let $\left\{P_{i}\right\}$ be a maximal set of mutually orthogonal central projections such that for each $i$ there is a natural number $j(i)$ with $\left(F_{k}-F_{k+1}\right) P_{i} \in$ $\mathscr{F}$ whenever $k \geqq j(i)$. This means that $A F_{j(i)} P_{i} \in \mathscr{F}$ since 


$$
\left\|A F_{j(i)} P_{i}(\mathscr{J})\right\|=\left\|A F_{k} P_{i}(\mathscr{J})\right\| \leqq k^{-1}
$$

for arbitrary $k \geqq j(i)$. Hence, setting $F=\sum F_{j(i)} P_{i}$ and $P=\sum P_{i}$, we obtain a projection $F$ of central support $P$ such that $A F \in \mathscr{J}$ and $E P \prec F$ (Proposition 2.9). Since $\mathscr{A}$ has no finite type $I$ direct summands, we may find a sequence $\left\{G_{n}^{\prime}\right\}$ of orthogonal projections of sum $F P$ such that the central support of $G_{n}^{\prime}$ is $P$ and such that $E P \prec$ $G_{n}^{\prime}$. Indeed, there is a central projection $R$ majorized by $P$ such that $F R$ is properly infinite and $F(P-R)$ is finite. In the first instance $F R$ is the sum of a sequence of mutually orthogonal projections each equivalent to $F R$ [9; III, 8, Corollary 2]. In the second instance, we have that $E(P-R)=0$. Indeed, $E$ is a properly infinite projection and $E(P-R)$ is finite since $E(P-R) \prec F(P-R)$. Now $F(P-R)$ may be written as the sum of a sequence of orthogonal projections of central support $P-R$ [9; III, 1, Theorem 1, Corollary 3].

Now, for every nonzero central projection $Q$ majorized by $P^{\prime}=$ $1-P$ and for every $n=1,2, \cdots$, there is a nonzero central projection $Q^{\prime}$ with $Q^{\prime} \leqq Q$ and a natural number $m \geqq n$ such that $\left(F_{m}-\right.$ $\left.F_{m+1}\right) Q^{\prime}$ has central support $Q^{\prime}$ and $E Q^{\prime} \prec\left(F_{m}-F_{m+1}\right) Q^{\prime}$ (Proposition 2.9). By induction we may find sets $\left\{G_{n i} \mid i \in I_{n}\right\}(1 \leqq n<\infty)$ of projections with the following properties:

(1) if $Q_{n i}$ denotes the central support of $G_{n i}$, then $E Q_{n i} \prec G_{n i} Q_{n i}$ $\left(i \in I_{n} ; n=1,2, \cdots\right)$;

(2) $\left\{Q_{n i} \mid i \in I_{n}\right\}$ is a mutually orthogonal set of sum $P^{\prime}$;

(3) for each $i \in I_{n}$ there is a natural number $s=s(i) \geqq n$ with $G_{n i}=$ $\left(F_{s}-F_{s+1}\right) Q_{n i} ;$ and

(4) if $i \in I_{m}, j \in I_{n}$, and $Q_{m i} Q_{n j} \neq 0$ then $s(i)<s(j)$ whenever $m<n$. Here $I_{n}$ is a countable indexing set with $I_{m} \cap I_{n} \neq \varnothing$ for $m \neq n$. Indeed, at the $(n+1)$ - st stage of the induction we work in algebras of the form $\mathscr{A} Q_{1 i_{i}} \cdots Q_{n i_{n}}\left(i_{j} \in I_{j}\right)$ and then sum the appropriate pieces together by summing over those pieces corresponding to the same $s(i)$. Setting $G_{n}^{\prime \prime}=\sum\left\{G_{n i} \mid i \in I_{n}\right\}$, we obtain sequence of mutually orthogonal projections of central support $P^{\prime}$ such that $E P^{\prime} \prec G_{n}^{\prime} P^{\prime}$, $A G_{n}^{\prime \prime}=G_{n}^{\prime \prime} A$, and $\left\|A G_{n}^{\prime \prime}\right\| \leqq n^{-1}$ for every $n=1,2, \cdots$. Setting $E_{n}=$ $G_{n}^{\prime}+G_{n}^{\prime \prime}$ for $n=1,2, \cdots$, we obtain a sequence $\left\{E_{n}\right\}$ of mutually orthogonal projections of central support 1 such that

$$
E \prec E_{n}, A E_{n}(\mathscr{F})=E_{n} A(\mathscr{F}), \text { and }\left\|A E_{n}(\mathscr{F})\right\| \leqq n^{-1}
$$

for every $n$.

Conversely, let $\left\{E_{n}\right\}$ be a sequence of (not necessarily orthogonal) projections which satisfy the conditions of the proposition for the central element $A_{0}$. Suppose there is a $B$ in $\mathscr{A}$ with $B\left(A_{0}-A\right)(\mathscr{F}(\zeta))=$ $1 \neq 0$ for some $\zeta$ in the spectrum of the center. Then we have 
that

$$
\left\|E_{n}(\mathscr{I}(\zeta))\right\|=\left\|B\left(A_{0}-A\right) E_{n}(\mathscr{F}(\zeta))\right\| \leqq n^{-1}\|B\|,
$$

for every $n=1,2, \cdots$ implies $\left\|E_{n}(\mathscr{J}(\zeta))\right\|=0$ for all sufficiently large $n$. However, this means that $\left\|E_{n}\left(\mathscr{\mathcal { J }}\left(\zeta^{\prime}\right)\right)\right\|=0$ for all $\zeta^{\prime}$ in a neighborhood of $\zeta$ since $\zeta^{\prime} \rightarrow\left\|E_{n}\left(\mathscr{J}\left(\zeta^{\prime}\right)\right)\right\|$ is a continuous function of the spectrum of the center into $\{0,1\}$ (Theorem 3.2). So there is a projection $P$ in the center with $P^{\wedge}(\zeta)=1$ such that $E_{n} P \in \mathscr{J}$ (Lemma 3.1). But this contradicts the hypothesis that $\operatorname{dim} E_{n}>\operatorname{dim} \mathscr{F}$ (Proposition 2.9). Consequently, the element $A_{0}$ is in the essential spectrum of $A$ with respect to $\mathscr{F}$.

CoROLlary 3.15. Let $\mathscr{A}$ be a von Neumann algebra with no finite type I direct summands and let $\mathscr{S}$ be a central ideal of $\mathscr{A}$. Then the essential central spectrum with respect to $\mathscr{F}$ of a self-adjoint element $A$ contains $A_{0}$ if and only if there is a sequence $\left\{E_{n}\right\}$ of mutually orthogonal projections of dimension greater than $\operatorname{dim} \mathscr{I}$ such that $\left\|\left(A_{0}-A\right) E_{n}\right\| \leqq n^{-1}$ for every $n=1,2, \cdots$ and $A_{0}=A_{0} P_{-}$.

Proof. There is no loss of generality in the assumption that $A_{0}=0$ since every element in the essential central spectrum of $A$ is selfadjoint. Then there are orthogonal projections $\left\{F_{n}\right\}$ such that $\operatorname{dim} F_{n}>$ $\operatorname{dim} \mathscr{F}, A F_{n}(\mathscr{J})=F_{n} A(\mathscr{F})$ and $\left\|A F_{n}(\mathscr{J})\right\|<(2 n)^{-1}$ for every $n=$ $1,2, \cdots$ (Proposition 3.14). For every $n$ there is a $B_{n} \in \mathscr{J}$ with $\left\|A F_{n}-B_{n} F_{n}\right\|<(2 n)^{-1}$. There is a projection $G_{n} \in \mathscr{J}$ such that $G_{n} \leqq$ $F_{n}$ and $\left\|B_{n} F_{n}\left(1-G_{n}\right)\right\| \leqq(2 n)^{-1}$. Let $E_{n}=F_{n}-G_{n}$. If $Q$ is a central projection with $Q E_{n} \in \mathscr{F}$, then $Q F_{n} \in \mathscr{F}$ and $Q P_{.}=0$ and so $\operatorname{dim} E_{n}>$ $\operatorname{dim} \mathscr{F}$ (Proposition 2.9.). But we have that

$$
\left\|A E_{n}\right\| \leqq\left\|\left(A-B_{n}\right) E_{n}\right\|+\left\|B_{n} E_{n}\right\| \leqq n^{-1} .
$$

Thus $\left\{E_{n}\right\}$ is the required sequence.

The converse is derived from Proposition 3.14 since $\|B(\mathscr{F})\| \leqq$ $\|B\|$ for every $B \in \mathscr{A}$.

CoROLlaRy 3.16. Let $\mathscr{A}$ be a von Neumann algebra with no finite type I direct summand and let $\mathscr{J}$ be a central ideal in $\mathscr{A}$. If the left-essential (resp. right-essential) central spectrum of an element $A$ in $\mathscr{A}$ contains $A_{0}$, then there is a sequence $\left\{E_{n}\right\}$ of orthogonal projections in $\mathscr{A}$ such that $\operatorname{dim} E_{n}>\operatorname{dim} \mathscr{F}$ and $\left\|\left(A_{0}-A\right) E_{n}\right\| \leqq n^{-1}$ (resp. $\left.\left\|\left(A_{0}-A\right)^{*} E_{n}\right\| \leqq n^{-1}\right)$ for every $n=1,2, \cdots$.

Proof. Since the essential central spectrum of $\left(A_{0}-A\right)^{*}\left(A_{0}-A\right)$ contains 0 , the Corollary 3.15 can be applied. 
REMARK 3.17. If $\mathscr{A}$ is a finite type I algebra, $\mathscr{J}$ is a central ideal, $A \in \mathscr{A}$, and $A_{0}$ in the essential central spectrum of $A$ with respect to $\mathscr{F}$, then D. Deckard and C. Pearcy [6] showed that there is an abelian projection $E$ of central support $P_{\mathcal{F}}$ in $\mathscr{A}$ with $\left(A_{0}-A\right) E=$ 0 .

4. The essential central range. Let $\mathscr{A}$ be a von Neumann algebra with center $\mathscr{\varkappa}$. Then $\mathscr{A}$ may be considered as a module over $\mathscr{Z}$. Let $\mathscr{A}^{\sim}$ be the $\mathscr{Z}$-module of all bounded module homomorphisms of $\mathscr{A}$ into $\mathscr{Z}$ and let $\mathscr{A}^{\sim+}$ be the set of all elements of $\mathscr{A}^{\sim}$ which map $\mathscr{A}^{+}$into $\mathscr{Z}^{+}$. For a central ideal $\mathscr{J}$ of $\mathscr{A}$, let $E_{a}(\mathscr{J})=$ $\left\{\phi \in \mathscr{A}^{\sim+} \mid \phi(\mathscr{J})=(0)\right.$ and $\left.\phi\left(P_{\mathscr{f}}\right)=P_{\mathscr{S}}\right\}$. Here $P_{\mathscr{S}}$ is the orthogonal complement of the largest central projection in $\mathscr{F}$. We notice that $E_{a}(\mathscr{J})$ is the set of all states (i.e. elements $\phi$ of $\mathscr{A}^{\sim+}$ with $\phi(1)=1$ \} of $\mathscr{A}^{\sim}$ which vanish on $\mathscr{J}$ whenever $P_{\mathscr{S}}=1$, or equivalently, if $\mathscr{J}=\mathscr{J}_{P}(E)$ (Remark 2.6), whenever the central support of $E$ is equal to $P$ (Corollary 2.7). In particular, the set $E_{a}(\mathscr{F})$ is equal to the set of all states which vanish on $\mathscr{F}$ whenever $\mathscr{F}$ is the ideal generated by the set of all finite projections or $\mathscr{F}$ is the strong radical of a properly infinite von Neumann algebra (Examples 2.11 and 2.12). It is clear that $E_{a}(\mathscr{J})$ is compact in the topology of pointwise convergence on $\mathscr{A}$ where is $\mathscr{Z}$ taken with the weak topology, i.e., in the $\sigma_{w}(\mathscr{A} \sim \mathscr{A})$-topology of $\mathscr{A}^{\sim}$. If $\left\{\phi_{i} \mid i \in I\right\}$ is any subset of $E_{a}(\mathscr{J}\}$ and $\left\{P_{i} \mid i \in I\right\}$ is a set of orthogonal central projections of sum 1, then $\phi(A)=\sum P_{i} \phi_{i}(A)$ defines an element $\phi$ in $E_{a}(\mathscr{J})$. Furthermore, we see that $E_{a}(\mathscr{F})$ is central-convex in the sense that $C \phi_{1}+(1-C) \phi_{2}$ is in $E_{a}(\mathscr{J})$ for every $\phi_{1}$ and $\phi_{2}$ in $E_{a}(\mathscr{J})$ and $C$ in $\mathscr{Z}$ with $0 \leqq C \leqq 1$.

Definition 4.1. Let $\mathscr{A}$ be a von Neumann algebra, let $\mathscr{J}$ be a central ideal of $\mathscr{A}$, and let $A$ be an element of $\mathscr{A}$. The set $\mathscr{K}(A)=$ $\left\{\phi(A) \mid \phi \in E_{a}(\mathscr{\mathcal { S }})\right\}$ will be called the essential central range of $A$ with respect to $\mathscr{J}$. We notice that $\mathscr{K}_{\mathcal{S}}(A)$ is a central-convex, weakly compact (and consequently uniformly closed) subset of the sphere in the center of $\mathscr{A}$ of radius $\|A\|$ about the origin.

Proposition 4.2. Let $\mathscr{A}$ be a von Neumann algebra, let $\mathscr{J}$ be a central ideal of $\mathscr{A}$, and let $A$ be an element of $\mathscr{A}$. Then for every $\zeta$ in the spectrum of the center of $\mathscr{A}$, the set $\mathscr{K}_{\mathcal{F}}(A)(\zeta)=\left\{B^{\wedge}(\zeta) \mid B \in\right.$ $\left.\mathscr{K}_{\mathcal{F}}(A)\right\}$ is a compact set of complex numbers.

Proof. Since $\mathscr{K}_{\mathscr{Y}}(A)(\zeta)$ is bounded, it is sufficient to show that $\mathscr{C}_{\mathcal{Y}}(A)(\zeta)$ is closed. If $\alpha$ is the limit of a sequence $\left\{\phi_{n}(A)^{\wedge}(\zeta)\right\}$ where $\phi_{n} \in E_{a}(\mathscr{J})$ for every $n=1,2, \cdots$, we show that $\alpha \in \mathscr{K}_{\mathscr{F}}(A)(\zeta)$. There is no loss of generality in assuming that $\alpha=0$. We may assume 
that $\left|\phi_{n}(A)^{\wedge}(\zeta)\right|<n^{-1}$ for every $n=1,2, \cdots$. There is a sequence $\left\{P_{n}\right\}$ of central projections with $\left\|\phi_{n}(A) P_{n}\right\| \leqq n^{-1}$, and $P_{n}^{\wedge}(\zeta)=1$ for every $n=1,2, \cdots$. Let $Q_{0}=\operatorname{glb}\left\{P_{1} \cdots P_{n} \mid n=1,2, \cdots\right\}$ and let $Q_{1}=$ $P_{1}\left(1-P_{2}\right), Q_{2}=P_{1} P_{2}\left(1-P_{3}\right), \cdots$; then $\left\{Q_{i} \mid i=0,1,2, \cdots\right\}$ is a sequence of orthogonal central projections of $\operatorname{sum} P_{1}$. The homomorphism

$$
\psi_{n}=\left(1-P_{1}\right) \phi_{1}+Q_{0} \phi_{n}+\sum\left\{Q_{i} \phi_{i} \mid i=1,2, \cdots\right\}
$$

is an element of $E_{a}(\mathscr{J})$ and so

$$
A_{0}=\lim \psi_{n}(A)=\left(1-P_{1}\right) \phi_{1}(A)+\sum\left\{Q_{i} \phi_{i}(A) \mid i=1,2, \cdots\right\}
$$

is in $\mathscr{K}(A)$. Since $\left(1-P_{1}\right)^{\wedge}(\zeta)=0$ and $Q_{i}^{\wedge}(\zeta)=0$ for all $i \geqq 1$, either $Q_{0}^{\wedge}(\zeta)=1$ or $\sum\left\{Q_{i} \mid i \geqq n\right\}^{\wedge}(\zeta)=1$ for all $n=1,2, \cdots$. In either case $A_{0}^{\wedge}(\zeta)=0$ since $\left\|\sum\left\{Q_{i} \mid i=0, n, n+1, \cdots\right\} A_{0}\right\| \leqq n^{-1}$. This means that $0 \in \mathscr{K}_{\zeta}(A)(\zeta)$.

We need the following lemma. Its proof is a simple reworking of [19; proof of corollary to (a5) implies (a1)].

Lemma 4.3. Let $\mathscr{A}$ be a von Neumann algebra, let $\mathscr{J}$ be a central ideal of $\mathscr{A}$, and let $E$ be a projection in $\mathscr{A}$. There is positive module homomorphism of the module $\mathscr{A}$ into its center which vanishes on $\mathscr{F}$ and satisfies the relation $\dot{\phi}(1)=\dot{\phi}(E)=1-Q$ where $Q$ is the largest central projection of $\mathscr{A}$ such that $E Q \in \mathscr{J}$.

Theorem 4.4. Let $\mathscr{A}$ be a von Neumann algebra. The essential central range of a self-adjoint element $A$ of $\mathscr{A}$ with respect to a central ideal $\mathscr{J}$ is the smallest central-convex subset of $\mathscr{A}$ which contains the essential central spectrum of $A$ with respect to $\mathscr{F}$.

Proof. Let $\mathscr{Z}$ be the center of $\mathscr{A}$, let $\zeta$ be in the spectrum of $\mathscr{Z}$, and let $\phi$ be an element of $E_{a}(\mathscr{F})$. Let $\phi_{\zeta}$ be the bounded linear functional on $\mathscr{A}$ defined by $\phi_{\zeta}(B)=\phi(B)^{\wedge}(\zeta)$ for all $B \in \mathscr{A}$. If $B_{1}, \cdots, B_{n}$ are in $\mathscr{A}$ and $C_{1}, \cdots C_{n}$ are in $\zeta$, then

$$
\phi_{\zeta}\left(\sum B_{i} C_{i}\right)=\sum C_{i}^{\wedge}(\zeta) \phi_{\zeta}\left(B_{i}\right)=0 .
$$

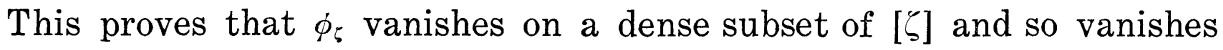

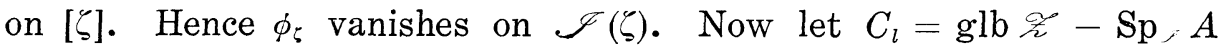
and $C_{u}=\operatorname{lub} \mathscr{\not}-\operatorname{Sp}_{\mathscr{C}} A$. We have that $C_{l}^{\wedge}(\zeta) \leqq A(\mathscr{F}(\zeta)) \leqq C_{u}^{\wedge}(\zeta)$ in $\mathscr{A}(\mathscr{S}(\zeta))$. (Proposition 3.12). This means that

$$
\phi\left(C_{l}\right)^{\wedge}(\zeta)=C_{\imath}^{\wedge}(\zeta) \leqq \phi(A)^{\wedge}(\zeta) \leqq C_{u}^{\wedge}(\zeta)=\phi\left(C_{u}\right)^{\wedge}(\zeta)
$$

for all $\zeta$ with $P_{\mathcal{G}}^{\wedge}(\zeta)=1$. Consequently, we have that $C_{l}=C_{l} P_{\mathcal{S}} \leqq$ $\phi(A) \leqq C_{u} P_{\mathcal{S}}=C_{u}$. So we may find a $C$ in $\not \mathcal{~ w i t h ~} 0 \leqq C \leqq 1$ such 
that $C C_{l}+(1-C) C_{u}=\phi(A)$. Hence, the smallest central convex set containing $\mathscr{Z}-\operatorname{Sp}_{\mathscr{I}} A$ contains $\mathscr{K}_{\mathcal{I}}(A)$.

Conversely, to show the opposite relation we simply must show that $C_{l}$ and $C_{u}$ are in $\mathscr{K}_{\mathscr{q}}(A)$. We work with $C_{u}$. Given $\varepsilon>0$, there is a projection $E$ in $\mathscr{A}$ such that $E$ commutes with $A,\left\|\left(C_{u}-A\right) E\right\|<$ $\varepsilon$, and if $E P$ is in $\mathscr{J}$ for a central projection $P$ then $P$ is in $\mathscr{J}$ (Proposition 3.13). There is a $\phi$ in $E_{a}(\mathscr{J})$ such that $\phi(E)=P_{\mathscr{S}}$ (Lemma 4.3). From the Cauchy-Schwarz inequality for elements of $A^{\sim+}$, we obtain

$$
\begin{aligned}
& \left\|\phi(A)-C_{u}\right\|=\left\|\phi\left(A-C_{u}\right)\right\|= \\
& \left\|\phi\left(\left(A-C_{u}\right) E\right)\right\|+\left\|\phi\left(\left(A-C_{u}\right)(1-E)\right)\right\| \\
& \leqq \varepsilon+\left\|A-C_{u}\right\|\|\phi(1-E)\|=\varepsilon .
\end{aligned}
$$

Because $\mathscr{K}_{\mathcal{C}}(A)$ is uniformly closed and because $\varepsilon>0$ is arbitrary, we have that $C_{u} \in \mathscr{K},(A)$. By a similar argument $C_{l} \in \mathscr{K}_{.}(A)$.

Corollary 4.5. Let $\mathscr{A}$ be a von Neumann algebra. The essential central range of an element $A$ in $\mathscr{A}$ with respect to a central ideal $\mathscr{F}$ is equal to a set $\left\{A_{0}\right\}$ if and only if $A_{0} P_{\mathscr{S}}=A_{0}$ and $A-A_{0} \in$ $\mathscr{F}$

Proof. First let the essential central range $\mathscr{K}_{\digamma}(A)$ of $A$ be equal to $A_{0}$. Then $\phi(A)=A_{0}$ for every $\phi \in E_{a}(\mathscr{J})$. Hence $\phi\left(A+A^{*}\right)=$ $A_{0}+A_{0}^{*}$ for every $\phi \in E_{a}(\mathscr{F})$. This means that the essential central spectrum of $A+A^{*}-\left(A_{0}+A_{0}^{*}\right)$ with respect to the ideal $\mathscr{J}$ is equal to $\{0\}$ (Theorem 4.4). Hence $A+A^{*}-\left(A_{0}+A_{0}^{*}\right) \in \mathscr{I}$ (Proposition 3.12 and Lemma 3.1). Similarly we find that $\left(A-A^{*}\right)-\left(A_{0}-A_{0}^{*}\right) \in \mathscr{F}$. Consequently, we have that $A-A_{0} \in \mathscr{F}$.

The converse is obvious.

The following remarks lead to a characterization of the essential central range. This reduces to the known characterization of the essential numerical range of the algebra of all bounded operators on a separable Hilbert space $[11 ; 5.1]$. Let $\mathscr{A}$ be a von Neumann algebra on the Hilbert space $H$ and let $\mathscr{\varkappa}$ be the center of $\mathscr{A}$. Let $E$ be an abelian projection with central support $P$ in the commutant $\mathscr{Z}^{\prime}$ of $\mathscr{Z}$ [cf. 9; I, §7]. For every $A \in \mathscr{A}$, there is a unique $\tau_{E}(A)$ in $\mathscr{Z} P$ with $E A E=\tau_{E}(A) E$. Then $A \rightarrow \tau_{E}(A)$ defines an element in $\mathscr{A}^{\sim+}$ with $\tau_{E}(1)=P$. For every projection $P$ in $\not 2$ let $V_{P}(\mathscr{A})=\left\{\tau_{E} \in\right.$ $\mathscr{A}^{\sim} \mid E$ is an abelian projection in $\mathscr{L}^{\prime \prime}$ of central support $\left.P\right\}$; for every $A \in \mathscr{A}$ let $W_{P}(A)=$ uniform closure $\left\{\phi(A) \mid \dot{\phi} \in V_{P}(\mathscr{A})\right\}$.

We now need a version of the Toeplitz-Hausdorff Theorem.

Lemma 4.6. Let $\mathscr{A}$ be a von Neumann algebra. Then, for 
every $A \in \mathscr{A}$ and central projection $P$, the set $\left\{\phi(A) \mid \phi \in V_{P}(\mathscr{A})\right\}$ is central-convex.

Proof. There is no loss of generality in the assumption that $P=1$. Let $E_{1}$ and $E_{2}$ be maximal abelian projections (i.e. abelian projections with central support 1 ) in the commutant $\mathscr{Z}^{\prime}$ of the center $\mathscr{z}$ of $\mathscr{A}$ and let $C \in \mathscr{Z}$ with $0 \leqq C \leqq 1$. Setting $E=\operatorname{lub}\left\{E_{1}, E_{2}\right\}$, we obtain a projection $E$ such that the reduced algebra $\mathscr{Z}_{E}^{\prime}$ is the product of homogeneous algebras of type $I_{n}$ where $n \leqq 2$. Indeed, we have that $\operatorname{lub}\left\{E_{1}, E_{2}\right\}-E_{1} \prec E_{1}$ and so $\operatorname{lub}\left\{E_{1}, E_{2}\right\}-E_{1}$ is abelian. So there is no loss of generality in the assumption that $\mathscr{A}=\mathscr{C}^{\prime}$ is homogeneous of degree 2 since the degee 1 case requires no further proof. Now we may write $\phi(B)=C \tau_{E_{1}}(B)+(1-C) \tau_{E_{2}}(B)$ as $\phi(B)=$ $A_{1} \tau_{F_{1}}(B)+A_{2} \tau_{F_{2}}(B)$ where $F_{1}, F_{2}$ are orthogonal maximal abelian projections of sum 1 and $A_{1}, A_{2}$ are elements in $\mathscr{2}^{+}$with $A_{1}+A_{2}=1$ [14; $\S 4$ ]. So we may assume that $E_{1}$ and $E_{2}$ are orthogonal of sum 1 . Let $\tau_{i}=\tau_{E_{i}}(i=1,2)$. Since it is sufficient to find a maximal abelian projection $E$ with $\tau_{E}\left(A-\tau_{2}(A)\right)=C \tau_{1}\left(A-\tau_{2}(A)\right)$, we may assume that $\tau_{2}(A)=$ 0 . Now there is a sequence $\left\{P_{n}\right\}$ of orthogonal projections in $\not{z}$ such that $\tau_{1}(A) P_{n}$ is invertible in $\mathscr{\mathcal { L }} P_{n}$ and $\tau_{1}(A)\left(1-\sum P_{n}\right)=0$. Because the sum of abelian projections with orthogonal central supports is again abelian, there is no loss of generality in the assumption that $\tau_{1}(A)=1$.

The rest of this lemma is the classical Toeplitz-Hausdorff theorem. Let $U$ be a partial isometry of $\mathscr{A}$ with $U^{*} U=E_{1}$ and $U U^{*}=E_{2}$ and let $A=E_{1}+A_{1} U+A_{2} U^{*}$, where $A_{1}, A_{2} \in \mathscr{\%}$. There is a unitary operator $V$ in $\mathscr{z}$ with $V\left|A_{1}-A_{2}^{*}\right|=A_{1}-A_{2}^{*}$. Let $T=V^{*} A_{1}+V A_{2}$. There is a $D \in \mathscr{Z}$ with $-1 \leqq D \leqq 1$ such that

$$
D^{2}+D\left(1-D^{2}\right)^{1 / 2} T=C
$$

[6]. Now, by direct calculation, we find that

$$
E=D^{2} E_{1}+V D\left(1-D^{2}\right)^{1 / 2} U+V^{*} D\left(1-D^{2}\right)^{1 / 2} U^{*}+\left(1-D^{2}\right) E_{2}
$$

is a projection in $\mathscr{A}$ of central support 1 that vanishes on the range of $\left(1-C^{2}\right)^{1 / 2} E_{1}-V C E_{2}$. So $E$ must be a maximal abelian projection. Finally, by another calculation, we obtain that $E A E=C E$.

Let $\mathscr{A}$ be a von Neumann algebra with center $\mathscr{z}$. Let $\mathscr{A}$ be considered as a $\mathscr{Z}$-module and let $\mathscr{A} \sim$ be the $\mathscr{Z}$-module of all $\sigma$-weakly continuous module homomorphisms of $\mathscr{A}$ into $\mathscr{Z}$. Let $\mathscr{A}_{\sim}^{+}=\mathscr{A}^{++} \cap \mathscr{A}_{\sim}$ be the set of all normal (i.e. positive $\sigma$-weakly continuous) module homomorphisms of $\mathscr{A}$ into $\mathscr{z}$.

Now we can extend Lemma 4.6. 
Lemma 4.7. Let $\mathscr{A}$ be a von Neumann algebra, let $P$ be a central projection of $\mathscr{A}$, and let $A \in \mathscr{A}$; then

$$
W_{P}(A)=\left\{\phi(A) \mid \phi \in \mathscr{A}_{\sim}^{+}, \phi(1)=P\right\} .
$$

Proof. First let $\phi \in \mathscr{A}_{\sim}^{+}$with $\phi(1)=P$. We show that $\phi(A) \in$ $W_{P}(A)$. There is a monotonely decreasing sequence $\left\{A_{n}\right\}$ of positive elements in the center $\mathscr{F}$ of $\mathscr{A}$, and a sequence $\left\{E_{n}\right\}$ of orthogonal abelian projections in the commutant $\mathscr{z}$ ' of $\mathscr{Z}$ with central supports $\left\{P_{n}\right\}$ respectively such that $\lim A_{n}=0$ (uniformly), $\sum A_{n}=P$ (strongly), $E_{n+1} \prec E_{n}, \operatorname{supp} P_{n}^{\wedge}=\operatorname{supp} A_{n}^{\wedge} \quad(n=1,2, \cdots)$, and $\phi(B)=\sum A_{n} \tau_{E_{n}}(B)$ (strongly) for all $B \in \mathscr{A}$ ([16; Theorem 2] and [14; $\$ 4])$. There is a mutually orthogonal set $\left\{Q_{i}\right\}$ in $(\mathscr{z})$ of $\operatorname{sum} P$ such that

$$
\lim _{m} \sum\left\{A_{n} Q_{i} \mid 1 \leqq n \leqq m\right\}=P Q_{i}
$$

uniformly (cf. [14, Theorem 4.1]). For each $Q_{i}$ we may therefore find an $m_{i}$ with $\left\|B_{i} Q_{i}\right\| \leqq \varepsilon$, where $B_{i}=\sum\left\{A_{n} \mid n \geqq m_{i}\right\}$ and where $\varepsilon>0$ is a preassigned constant. Now there are abelian projections $F_{k}(1 \leqq$ $k \leqq m_{i}=m$ ) of central support $P Q_{i}$ such that $E_{k} Q_{i} \leqq F_{k}$. Since $\operatorname{supp} P_{k}^{\wedge}=\operatorname{supp} A_{k}^{\wedge}$, we have that $\phi_{i}=\sum\left\{A_{k} \tau_{F_{k}} \mid 1 \leqq k \leqq m-1\right\}+B_{i} \tau_{F_{m}}$ is equal to $\sum\left\{A_{k} Q_{i} \tau_{E_{k}} \mid 1 \leqq k \leqq m-1\right\}+B_{i} \tau_{F_{m}}$. Since $\sum\left\{A_{k} Q_{i} \mid 1 \leqq\right.$ $k \leqq m-1\}+B_{i} Q_{i}=P Q_{i}$, there is an abelian projection $G_{i}$ in $\mathcal{z}^{\prime \prime}$ of central support $P Q_{i}$ such that $\tau_{G_{i}}(A)=\phi_{i}(A)$ (Lemma 4.6). Notice that

$$
\left\|\left(\phi_{i}(A)-\phi(A)\right) Q_{i}\right\| \leqq\left\|B_{i} \tau_{F_{m}}(A)\right\|+\left\|\sum\left\{A_{n} \tau_{E_{n}}(A) \mid n \geqq m\right\} Q_{i}\right\| \leqq 2 \varepsilon\|A\| \cdot
$$

Now $\sum G_{i}=G$ is an abelian projection of central support $P$ and

$$
\left\|\dot{\phi}(A)-\tau_{G}(A)\right\| \leqq \operatorname{lub}\left\|\left(\phi(A)-\tau_{G_{i}}(A)\right) Q_{i}\right\| \leqq 2 \varepsilon\|A\| \text {. }
$$

So $\phi(A) \in W_{P}(A)$ since $\varepsilon>0$ is arbitrary and $W_{P}(A)$ is closed.

The converse relation is obvious since $\tau_{E}$ is a normal module homomorphism.

Proposition 4.8. Let $\mathscr{A}$ be a von Neumann algebra. Then the essential central range of an element $A$ in $\mathscr{A}$ with respect to the central ideal $\mathscr{F}$ is equal to $\cap\left\{W_{P}(A+B) \mid B \in \mathscr{J}\right\}$. Here $P=P_{\mathscr{C}}$.

Proof. Let $\dot{\phi} \in E_{a}(\mathscr{F})$. Let $Q$ be the central projection in $\mathscr{A}$ such that $\mathscr{A} Q$ is a discrete algebra and $\mathscr{A}(1-Q)$ is a continuous algebra. There is a net $\left\{\phi_{n}^{\prime}\right\}$ (resp. $\left.\left\{\phi_{m}^{\prime \prime}\right\}\right)$ of elements of $(\mathscr{A} Q)_{\sim}^{+}($resp. $\left.(\mathscr{A}(1-Q))_{\sim}^{+}\right)$with $\phi_{n}^{\prime}(Q)=P_{\mathcal{J}} Q$ (resp. $\left.\phi_{m}^{\prime \prime}(1-Q)=P_{\mathcal{S}}(1-Q)\right)$ such that $\lim \phi_{n}(B Q)=\phi(B Q)\left(\right.$ resp. $\left.\lim \phi_{m}(B(1-Q))=\phi(B(1-Q))\right)$ uniformly for every $B \in \mathscr{A}$. This follows from Theorem 5.4 (resp. Theorem 5.1) of [17]. Then setting $\phi_{n m}(B)=\phi_{n}^{\prime}(B Q)+\phi_{m}^{\prime \prime}(B(1-Q))$, we obtain a 
net $\left\{\phi_{n m}\right\}$ in $\mathscr{A}_{\sim}^{+}$with $\phi_{n m}(1)=P_{\mathscr{S}}$ for all $m, n$ and $\lim \phi_{n m}(B)=\phi(B)$ (uniformly) for all $B \in \mathscr{A}$. Let $B \in \mathscr{I}$ and let $\varepsilon>0$; then there is a $\phi_{m n}$ with $\left.\| \phi_{m n}(B)\right) \| \leqq \varepsilon$ and $\left\|\phi_{m n}(A)-\phi(A)\right\| \leqq \varepsilon$ since $\phi(B)=0$. Since $\varepsilon>0$ is arbitrary and since $W_{P}(A+B)$ is closed, we have that $\phi(A) \in W_{P}(A+B)$ by Lemma 4.7. Since $B \in \mathscr{J}$ is arbitrary $\phi(A) \in$ $\cap\left\{W_{P}(A+B) \mid B \in \mathscr{I}\right\}$. So $\mathscr{K}_{\mathcal{F}}(A) \subset \cap\left\{W_{P}(A+B) \mid B \in \mathscr{F}\right\}$.

We now prove that the opposite inclusion relation is true. First let $A$ be self-adjoint. We show that $0 \in \cap\left\{W_{P}(A+B) \mid B=B^{*} \in \mathscr{F}\right\}$ implies that $0 \in \mathscr{K} \mathcal{G}(A)$. Let $\mathscr{Z}$ be the center of $\mathscr{A}$ and let $C_{u}=$ lub $\mathscr{2}-\operatorname{Sp}_{\mathscr{A}} A$. Suppose there is an $\alpha>0$ and a nonzero projection $Q$ in $\mathscr{z}$ with $Q \leqq P$ and $C_{u} Q \leqq-2 \alpha Q$. We have that $\left(C_{u}-A\right)(\mathscr{F}(\zeta)) \geqq 0$ for every $\zeta$ in the spectrum of $\mathscr{F}$ (Proposition 3.12). If $f_{+}$(resp. $-f_{-}$) is the function that is identity on the real interval $[0, \infty)$ (resp. $(-\infty, 0])$ and 0 on the complement, we have that $f_{-}\left(C_{u}-A\right)$ is a self-adjoint element in $\mathscr{F}$ (Lemma 3.1). However, by hypothesis there is an abelian projection $E$ in $\mathscr{L}^{\prime}$ of central support $P$ with $\left\|\tau_{E}\left(f_{-}\left(C_{u}-A\right)-A\right)\right\| \leqq \alpha$. On the other hand, we have that

$$
Q \tau_{E}\left(f_{-}\left(C_{u}-A\right)-A\right)=Q \tau_{E}\left(f_{+}\left(C_{u}-A\right)-C_{u}\right) \geqq 2 \alpha Q .
$$

This is a contradiction. Hence, we find that $C_{u} P \geqq 0$. Since $1-P \in \mathscr{F}$, we have that $C_{u}(1-P)=0$ and so $C_{u} \geqq 0$ (cf. Remark 3.8). Similarly, we obtain $C_{l}=$ glb $\mathscr{\&}-\mathrm{Sp},(A) \leqq 0$ and finally that $0 \in \mathscr{K}_{,}(A)$ (Theorem 4.4).

Now let $A$ be an arbitrary element of $\mathscr{A}$ with $0 \in \cap\left\{W_{P}\{A+\right.$ $B) \mid B \in \mathscr{F}\}$. Let $\mathscr{S}=\left\{|B|=\left(B^{*} B\right)^{1 / 2} \mid B \in \mathscr{K}(A)\right\}$. We note that $\mathscr{S}$ is a monotonely decreasing net in $\mathcal{O}^{+}$. Indeed, let $B$ and $C$ be in $\mathscr{K}_{5}(A)$. There is a central projection $Q$ with $Q|B|+(1-Q)|C|=$ $\operatorname{glb}\{|B|,|C|\}$. But the set $\mathscr{K}_{\mathcal{Y}}(A)$ is central-convex and so $\mathscr{K}_{\mathcal{S}}(A)$ contains $D=Q B+(1-Q) C$. Thus, we have that $|D|=Q|B|+$ $(1-Q)|C|$ is in $\mathscr{S}$. Thus $\mathscr{S}$ has a greatest lower bound $B_{0}$ in $\mathscr{F}^{\circ+}$. We show $B_{0}=0$ by arguing by contradiction. Suppose there is a point $\zeta$ in the spectrum $Z$ of $\mathscr{Y}$ with $B_{0}^{\wedge}(\zeta)>0$. Then we may assume that $B_{0}^{\wedge}(\zeta)=\operatorname{glb}\left\{C^{\wedge}(\zeta) \mid C \in \mathscr{S}\right\}$ since $B_{0}^{\wedge}(\zeta)=\operatorname{glb}\left\{C^{\wedge}(\zeta) \mid C \in \mathscr{S}\right\}$ holds on a dense open set of $Z$ [7]. There is a $C \in \mathscr{K}_{\digamma}(A)$ such that $\left|C^{\wedge}(\zeta)\right|=$ $B_{0}^{\wedge}(\zeta)$ (Proposition 4.2). Then we may find a unitary $U$ in $\mathscr{F}$ such that $U C=|C|$. We have that $0 \in \cap\left\{W_{P}(U A+B) \mid B \in \mathscr{J}\right\}$ since $U\left(W_{P}\left(A+U^{*} B\right)\right)=W_{P}(U A+B)$ and that $B_{0}=\operatorname{glb}\left\{|B| \mid B \in \mathscr{K}_{\mathcal{S}}(U A)\right\}$ since $\mathscr{K}_{,}(U A)=U \mathscr{K}_{\mathcal{Y}}(A)$. Furthermore, we have that $|C| \in \mathscr{K}_{\mathscr{\digamma}}(U A)$. Hence, there is no loss in generality in assuming that there is a $C \in$ $\mathscr{K}_{\mathcal{Y}}(A)$ with $C^{\wedge}(\zeta)=B_{0}^{\wedge}(\zeta)$. Now let $A_{1}=\left(A+A^{*}\right) / 2$ and $A_{2}=(A-$ $\left.A^{*}\right) / 2 i$. We show that $0 \in \cap\left\{W_{P}\left(A_{j}+B\right) \mid B=B^{*} \in \mathscr{J}\right\}(1 \leqq j \leqq 2)$. In fact, given $\varepsilon>0$ and $B=B^{*} \in \mathscr{I}$, there is an abelian projection $E$ 
with central support $P$ in the commutant of $\mathscr{\digamma}$ such that $\left\|\tau_{E}(A+B)\right\| \leqq$ $\varepsilon$. Hence, we have that

$$
\left\|\tau_{E}\left(A+A^{*}+2 B\right)\right\|=\left\|\tau_{E}(A+B)+\tau_{E}(A+B)^{*}\right\| \leqq 2 \varepsilon .
$$

Similarly, we may find an abelian projection $F$ of central support $P$ such that $\left\|\tau_{F}\left(A-A^{*}+2 i B\right)\right\| \leqq 2 \varepsilon$. Now by the preceding paragraph we conclude that $0 \in \mathscr{K}_{\xi}\left(A_{j}\right)(1 \leqq j \leqq 2)$. Let $\phi$ be an element of $\mathscr{A}^{\sim+}$ with $\phi(1)=P, \phi(\mathscr{J})=0$, and $\phi\left(A_{1}\right)=0$. However, every element of the form $\alpha \phi(A)+(1-\alpha) C(0 \leqq \alpha \leqq 1)$ is in $\mathscr{K}_{S}(A)$ and so there is at least one $\alpha$ with $0 \leqq \alpha \leqq 1$ such that

$$
\left|\alpha \phi(A)^{\wedge}(\zeta)+(1-\alpha) C^{\wedge}(\zeta)\right|<C^{\wedge}(\zeta)=B_{0}^{\wedge}(\zeta) .
$$

Indeed $\phi(A)^{\wedge}(\zeta)$ is pure imaginary. This contradicts the choice of $B_{0}$. Hence, we must have that $0 \in \mathscr{K}_{\mathcal{F}}(A)$.

Proposition 4.9. Let $\mathscr{A}$ be a von Neumann algebra; then $A_{0}$ is in the essential central range of $A \in \mathscr{A}$ with respect to the central ideal $\mathscr{J}$ if $A_{0} P_{S}=A_{0}$ and if, given $\varepsilon>0$, there is a projection $E$ with $\operatorname{dim} E>\operatorname{dim} \mathscr{J}$ such that $\left\|E\left(A_{0}-A\right) E\right\| \leqq \varepsilon$. Conversely, if $A \in \mathscr{A}$ is self-adjoint and if $A_{0}$ is in the essential central range of $A$ with respect to $\mathscr{F}$, then there is a projection $E$ in $\mathscr{A}$ with $\operatorname{dim} E>$ $\operatorname{dim} \mathscr{F}$ such that $\left\|E\left(A_{0}-A\right) E\right\| \leqq \varepsilon$.

Proof. The first statement follows from Lemma 4.3 and Proposition 2.9 since the essential central range $\mathscr{K}_{\mathcal{F}}(A)$ of $A$ with respect to $\mathscr{F}$ is uniformly closed.

Now let $A$ be self-adjoint and let $A_{0} \in \mathscr{K}_{\mathcal{S}}(A)$. There is no loss of generality in assuming at the outset that $A_{0}=0$ and that $P_{S}=1$. Let $\mathscr{J}$ have the canonical form $\mathscr{I}=\mathscr{I}_{P}(F)$ (Remark 2.6). Let $\varepsilon>0$ be given. Let $C_{l}=\operatorname{glb} \mathscr{Z}-\mathrm{Sp}_{\mathscr{S}} A$ and let $C_{u}=\operatorname{lub} \mathscr{\not}-\mathrm{Sp}_{\mathcal{S}} A$ where $\mathscr{L}$ is the center of $\mathscr{A}$. Since $0 \in \mathscr{K}_{\mathcal{l}}(A)$, we have that $C_{l} \leqq$ $0 \leqq C_{u}$ (Theorem 4.4).

Now let $R$ be the largest central projection such that $\mathscr{A} R$ is of type $I$ and $\mathscr{J} R=0$. Consequently, if $G$ is a finite type $I$ projection majorized by $1-R$, then $G \in \mathscr{I}(1-R)$ (Proposition 2.2). By Proposition 3.10 we may assume that either $R=1$ or $1-R=1$.

First suppose theat $R=1$. We may assume that $\mathscr{A}$ is equal the commutant of its center [9; I, 8, Theorem 1]. Then there are abelian projections $E_{1}$ and $E_{2}$ of central support 1 in $\mathscr{A}$ such that

$$
\left\|\tau_{E_{1}}(A)-C_{l}\right\|+\left\|\tau_{E_{2}}(A)-C_{u}\right\| \leqq \varepsilon .
$$

(Theorem 4.4 and Proposition 4.8). There is a $C$ in $\mathscr{Z}$ with $0 \leqq C \leqq 1$ such that $C C_{l}+(1-C) C_{u}=0$, and there is an abelian projection $E$ 
of central support 1 in $\mathscr{A}$ such that $\tau_{E}(A)=C \tau_{E_{1}}(A)+(1-C) \tau_{E_{2}}(A)$ (Lemma 4.6). Thus, we obtain

$$
\|E A E\| \leqq\|C\|\left\|\tau_{E_{1}}(A)-C_{l}\right\|+\|1-C\|\left\|\tau_{E_{2}}(A)-C_{u}\right\| \leqq \varepsilon .
$$

So we may assume that $1-R=1$. Because the closure of every open subset of the spectrum $Z$ of $\mathscr{Z}$ is open, we may find a sequence $\left\{P_{n} \mid n=0,1,2, \cdots\right\}$ of mutually orthogonal central projections of sum 1 such that

$$
C_{l} P_{n} \leqq-n^{-1} P_{n}<0<n^{-1} P_{n} \leqq C_{u} P_{n}
$$

for $n=1,2, \cdots$, and $C_{l} C_{u} P_{0}=0$. We shall find projections $E_{n}$ of central support $P_{n}$ such that $F P_{n} \prec E_{n}$ and $\left\|E_{n} A E_{n}\right\| \leqq 4 \varepsilon$. Then we shall have that $E=\sum E_{n}$ has central support $1, F \prec E$, and $\|E A E\|=$ $\operatorname{lub}_{n}\left\|E_{n} A E_{n} P_{n}\right\| \leqq 4 \varepsilon$ (cf. [9, III, $\left.\left.\S 1\right]\right)$. Now, we have that $\mathscr{I}_{n}=$ $\mathscr{I} P_{n}=\mathscr{I}_{P P_{n}}\left(E P_{n}\right)$ is a representation of the central ideal $\mathscr{F}_{n}$ of $\mathscr{A} P_{n}$ in canonical form. Since $C_{l} C_{u} P_{0}=0$, there is a $P_{0}^{\prime}$ in $\left(\mathscr{z} P_{0}\right)$ with $P_{0}^{\prime} C_{l}+\left(P_{0}-P_{0}^{\prime}\right) C_{u}=0$ (Lemma 3.4). Thus, we see that $0 \in \mathscr{Z} P_{0}-$ $\operatorname{Sp}_{\mathcal{F}_{0}}\left(A P_{n}\right)$ (Proposition 3.10) and so we may find the projection $E_{0}$ (Proposition 3.13). By reducing to an algebra $\mathscr{A} P_{n}$, we may assume that $C_{l} \leqq-\alpha<0<\alpha \leqq C_{u}$ (Proposition 3.10).

It is sufficient to show that every nonzero $Q \in(\mathscr{L})$ majorizes a nonzero $R \in(\mathscr{Z})$ such that there is a $G \in(\mathscr{A})$ of central support $R$ with $F R \prec G$ and $\|G A G\| \leqq 4 \varepsilon$. Then the usual maximality argument for the projections $R$ may be employed to find the projection $E_{n}$. By making yet another reduction to a direct summand of $\mathscr{A}$, we may assume, without loss of generality, that there are natural numbers $m, n$, and $p$ such that

$$
\left\|m p^{-1}+C_{l}\right\| \leqq p^{-1} \leqq \varepsilon \text { and }\left\|n p^{-1}-C_{u}\right\| \leqq \varepsilon .
$$

We now find $n$ (resp. $m$ ) orthogonal projections $F_{i}$ of dimension greater than $\operatorname{dim} \mathscr{F}$ such that $\left\|\left(C_{l}-A\right) F_{i}\right\| \leqq \varepsilon\left(\right.$ resp. $\left\|\left(C_{u}-A\right) F_{i}\right\| \leqq$ $\varepsilon)$. We normally would apply Proposition 3.14, however it is necessary for the combined set of $m+n$ projections to be orthogonal and so the following additional argument is required. Let $A_{1}$ and $A_{2}$ be elements of $\mathscr{A}^{+}$such that $A_{1}-A_{2}=A$ and $A_{1} A_{2}=0$. For every $\zeta \in$ $Z$, we have that $-C_{\imath}^{\wedge}(\zeta)=\left\|A_{2}(\mathscr{F}(\zeta))\right\|$ and $C_{u}^{\wedge}(\zeta)=\left\|A_{1}(\mathscr{I}(\zeta))\right\|$ (Proposition 3.12). Let $G_{1}$ and $G_{2}$ be the domain projections of $A_{1}$ and $A_{2}$, respectively. For definiteness, let $G=G_{1}$. If $Q$ is a central projection with $G Q \in \mathscr{F}$, then $Q=0$; otherwise, there is a $\zeta \in Z$ with $G(\mathscr{J}(\zeta))=G Q(\mathscr{J}(\zeta))=0$ and consequently with $\left\|A_{1}(\mathscr{J}(\zeta))\right\|=0$. This implies that $\operatorname{dim} G>\operatorname{dim} \mathscr{F}$. So there is a projection $G^{\prime}$ with $F \sim G^{\prime} \leqq G$. We now restrict $\mathscr{A}$ to the subspace of the Hilbert space determined by $G$ to obtain the von Neumann algebra $\mathscr{A}_{G}=$ 
$G \mathscr{A} G$ (cf. [9; I, §2]). The set $G \mathscr{\mathscr { F }} G=\mathscr{J}_{G}$ is easily seen to be a central ideal of $\mathscr{A}_{G}$ since the center of $\mathscr{A}_{G}$ is $\mathscr{\mathscr { Z }} G=\mathscr{\varkappa}_{G}$ [9; III, 5, Problem 7]. We have that $A_{1} \in \mathscr{A}_{G}^{+}$. Since the spectrum of $\mathscr{Z}_{G}$ is the set $X=\{\zeta G \mid \zeta \in Z\}$, we have that the smallest ideal $[\zeta G]$ of $\mathscr{A}_{G}$ which contains $\zeta G$ is $G[\zeta] G$. We may now easily show that

$$
\left\|A_{1}\left(\mathscr{I}_{G}+[\zeta G]\right)\right\|=\inf \left\{\|A+B+C\| \mid B \in \mathscr{I}_{G}, C \in[\zeta G]\right\}
$$

is equal to $\left\|A_{1}(\mathscr{J}(\zeta))\right\|$ for every $\zeta \in Z$. This means that $C_{u} G=$ lub $\mathscr{Z}_{G}-\operatorname{Sp}_{\mathscr{F}_{G}} A_{1}$ (Proposition 3.12). Therefore, we may find a set $F_{1}, F_{2}, \cdots, F_{m}$ of mutually orthogonal projections in $\mathscr{A}_{G}$ of dimension greater than $\operatorname{dim} \mathscr{I}_{G}$ such that

$$
A_{1} F_{i}\left(\mathscr{I}_{G}\right)=F_{i} A_{1}\left(\mathscr{F}_{G}\right) \text { and }\left\|\left(C_{u} G-A_{1}\right) F_{i}\left(\mathscr{F}_{G}\right)\right\| \leqq \varepsilon
$$

for every $i=1,2, \cdots, m$ (Proposition 3.14). Indeed, the algebra $\mathscr{A}_{G}$ has no finite type I direct summands. Thus, we may find orthogonal projections $F_{1}, F_{2}, \cdots, F_{m}$ majorized in $\mathscr{A}$ by $G=G_{1}$ such that

$$
F \sim G^{\prime} \prec F_{i}, A F_{i}(\mathscr{F})=F_{i} A(\mathscr{F}) \text {, and }\left\|\left(C_{u}-A\right) F_{i}(\mathscr{F})\right\| \leqq \varepsilon
$$

for every $i=1,2, \cdots, m$. Likewise, we may find orthogonal projections $F_{m+1}, \cdots, F_{m+n}$ majorized by $G_{2}$ such that $F \prec F_{i}, A F_{i}(\mathscr{J})=$ $F_{i} A(\mathscr{F})$, and $\left\|\left(C_{l}-A\right) F_{i}(\mathscr{J})\right\| \leqq \varepsilon$ for every $i=m+1, \cdots, m+n$. Since $G_{1}$ and $G_{2}$ are orthogonal, the projections $F_{1}, \cdots, F_{m+n}$ are mutually orthogonal. There are partial isometries $U_{i j}(1 \leqq i, j \leqq m+n)$ of $\mathscr{A}$ which satisfy the following properties:

(1) $U_{i j} U_{k l}=\delta_{i l} U_{k j}(\delta=$ Kronecker delta $)$;

(2) $U_{i j}=U_{j i}^{*}$; and

(3) $U_{i i}$ is a projection with $F \sim U_{i i} \prec F_{i}$, for all $i, j, k, l$. The element $E^{\prime}=(m+n)^{-1} \sum U_{i j}$ is a projection in $\mathscr{A}$ with $E^{\prime} \sim F$, i.e. $\operatorname{dim} E^{\prime}>\operatorname{dim} \mathscr{F}$. Here, indeed, a calculation using $(m+n) \times$ $(m+n)$ complex matrices suffices. Furthermore, using the fact that $A F_{i}(\mathscr{F})=F_{i} A(\mathscr{I})$ for every $i$, we have that

$$
\begin{aligned}
&\left\|E^{\prime} A E^{\prime}(\mathscr{F})\right\| \\
&=\left\|E^{\prime}\left(A-\left(C_{u} \sum\left\{U_{i i} \mid i \leqq m\right\}+C_{l} \sum\left\{U_{i i} \mid i>m\right\}\right)\right) E^{\prime}(\mathscr{F})\right\| \\
&+\left\|E^{\prime}\left(\sum\left\{C_{u} U_{i i} \mid i \leqq m\right\}+\sum\left\{C_{l} U_{i i} \mid i>m\right\}\right) E^{\prime}(\mathscr{I})\right\| \\
& \leqq \|(m+n)^{-2} \sum_{i j}\left(\sum\left\{U_{k i}\left(A-C_{u}\right) U_{j k} \mid k \leqq m\right\}\right. \\
&\left.+\sum\left\{U_{k i}\left(A-C_{l}\right) U_{j k} \mid k>m\right\}\right)(\mathscr{J}) \| \\
&+\left\|(m+n)^{-1}\left(m C_{u}+n C_{l}\right) E^{\prime}(\mathscr{F})\right\| \leqq 2 \varepsilon .
\end{aligned}
$$

Now there is a $B \in \mathscr{F}$ with $\left\|E^{\prime} A E^{\prime}-E^{\prime} B E^{\prime}\right\| \leqq 3 \varepsilon$. In the ideal $\mathscr{F}$, we may find a spectral projection $E^{\prime \prime}$ for $E^{\prime} B^{*} B E^{\prime}$ majorized by $E^{\prime}$ so that $\left\|B E^{\prime}\left(1-E^{\prime \prime}\right)\right\| \leqq \varepsilon$. If $Q\left(E^{\prime}-E^{\prime \prime}\right) \in \mathscr{J}$ for some $Q \in(\mathscr{Z})$, then $Q E^{\prime} \in \mathscr{F}$ and consequently $Q=0$. This means that 


$$
\operatorname{dim}\left(E^{\prime}-E^{\prime \prime}\right)>\operatorname{dim} \mathscr{F}
$$

(Proposition 2.9). Setting $E=E^{\prime}-E^{\prime \prime}$, we obtain the relation

$$
\|E A E\| \leqq\|E(A-B) E\|+\|E B E\| \leqq 4 \varepsilon .
$$

REMARK 4.10. If $\mathscr{A}$ is the algebra of all bounded operators on a separable Hilbert space $H$ and $\mathscr{J}$ is the ideal of completely continuous operators, then Fillmore, Stampfli, and Williams [11, Theorem 5.1, Corollary] have obtained Proposition 4.8 without the added restriction that $A$ is self-adjoint. The theorem of Fillmore, et al., depends on properties of Hilbert-Schmidt operators on separable $H$; however, it is likely that the restriction can also be removed here.

Let $\mathscr{A}$ be a von Neumann algebra. Let $U(\mathscr{A})$ be the group of unitary operators of $\mathscr{A}$ and let $\mathscr{E}$ be the set of positive real-valued functions $f$ of finite support such that $\sum\{f(U) \mid U \in U(\mathscr{A})\}=1$. For each $f \in \mathscr{E}$ and $A$ in $\mathscr{A}$, let $f \cdot A=\sum\left\{f(U) U^{*} A U \mid U \in U(\mathscr{A})\right\}$ and let $\mathscr{K}^{\prime}(A)$ be the uniform closure of $\{f \cdot A \mid f \in \mathscr{E}\}$. If $B \in \mathscr{K}^{\prime}(A)$, then $\mathscr{K}^{\prime}(B) \subset \mathscr{K}^{\prime}(A)$. Then the intersection $\mathscr{K}(A)$ of $\mathscr{K}^{\prime}(A)$ with the center is a nonvoid closed convex subset of the center ([8]; cf. also [9; III, §5]). Furthermore the set $\mathscr{K}^{\prime}(A)$ (resp. $\mathscr{K}(A)$ ) is centralconvex in the sense that $C C_{1}+(1-C) C_{2}$ is in $\mathscr{K}^{\prime}(A)$ (resp. $\left.\mathscr{K}^{\prime}(A)\right)$ for every $C_{1}$ and $C_{2}$ in $\mathscr{K}^{\prime}(A)$ (resp. $\mathscr{K}^{\prime}(A)$ ) and $C$ in the center with $0 \leqq C \leqq 1$ [19; proof, Lemma 6].

The following forms the basis for our analysis of $\varkappa^{\top}(A)$.

Proposition 4.11. Let $\mathscr{A}$ be a von Neuman algebra and let $A$ be an element in $\mathscr{A}$. Let $\zeta$ be a point in the spectrum of the center of $\mathscr{A}$. Then the set $\mathscr{K}(A)(\zeta)=\left\{B^{\wedge}(\zeta) \mid B \in \mathscr{K}(A)\right\}$ is a compact subset of the complex plane.

Proof. Because $\mathscr{\mathscr { C }}(A)(\zeta)$ is bounded, it is sufficient to show that $\mathscr{K}(A)(\zeta)$ contains an arbitrary limit point $\alpha$. Due to the fact that $\mathscr{K}(A-\alpha)(\zeta)=\mathscr{K}(A)(\zeta)-\alpha$, there is no loss in generality in proving that $0 \in \mathscr{K}(A)(\zeta)$ whenever 0 is a limit point of $\mathscr{K}(A)(\zeta)$. We proceed to do this. For every $n=1,2, \cdots$, there is a function $f_{n}$ in the subset $\mathscr{E}$ of real-valued functions on the unitary operators of $\mathscr{A}$ and a central projection $P_{n}$ of $\mathscr{A}$ with $P_{n}^{\wedge}(\zeta)=1$ and $\left\|\left(f_{n} \cdot A\right) P_{n}\right\| \leqq 2^{-n}$. Let $\left\{Q_{i}\right\}$ be the sequence of orthogonal projections defined by $Q_{1}=$ $P_{1}-P_{1} P_{2}, Q_{2}=P_{1} P_{2}-P_{1} P_{2} P_{3}, \cdots$, and let $B \in \mathscr{K}^{\prime}(A)$. Then let $C_{n}=$ $B\left(1-P_{1}\right)+\sum\left\{\left(f_{i} \cdot A\right) Q_{i} \mid 1 \leqq i \leqq n\right\}+\left(f_{n+1} \cdot A\right) Q_{n}^{\prime}(n=1,2, \cdots)$. Here $Q_{n}^{\prime}=P_{1} \cdots P_{n+1}$ is the orthogonal complement of $\left(1-P_{1}\right)+\sum\left\{Q_{i} \mid 1 \leqq\right.$ $i \leqq n\}$. We notice that $C_{n} \in \mathscr{K}^{\prime}(A)$ for every $n$ since $\mathscr{K}^{\prime}(A)$ is centralconvex. However, the sequence $\left\{C_{n}\right\}$ is Cauchy since $\left\|C_{n}-C_{n+1}\right\| \leqq$ $\max \left\{\left\|\left(f_{n+1} \cdot A\right) Q_{n+1}^{\prime}\right\|,\left\|\left(f_{n+2} \cdot A\right) Q_{n+1}^{\prime}\right\|\right\} \leqq 2^{-n-1}$. This means that $\left\{C_{n}\right\}$ con- 
verges to an element $C$ in $\mathscr{\varkappa}^{\prime \prime}(A)$. We have that

$$
\|C(\zeta)\|=\lim \left\|C_{n}(\zeta)\right\|=\lim \left\|\left(\left(f_{n+1} \cdot A\right) Q_{n}^{\prime}\right)(\zeta)\right\| \leqq \lim \sup 2^{-n}=0
$$

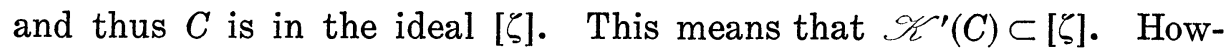
ever, we have that $\mathscr{K}^{\prime}(C) \subset \mathscr{K}^{\prime}(A)$ because $C \in \mathscr{K}^{\prime}(A)$. This means that $\mathscr{K}(A) \cap[\zeta] \neq \varnothing$, or equivalently, that $0 \in \mathscr{K}(A)(\zeta)$.

THEOREM 4.12. Let $\mathscr{A}$ be a properly infinite von Neumann algebra, let $\mathscr{J}$ be the strong radical of $\mathscr{A}$, and let $A$ be an element of $\mathscr{A}$. Then the set $\mathscr{K}_{(A)}\left(A\right.$ is equal to the set $\mathscr{K}_{\mathcal{Z}}(A)=\{\phi(A) \mid \phi$ is a state of $\mathscr{A}^{\sim}$ with $\left.\dot{\phi}(\mathscr{J})=(0)\right\}$.

REMARK 4.13. Here notice $E_{a}(\mathscr{J})$ is the set of all states of $\mathscr{A}^{\sim}$ which vanish on $\mathscr{J}$.

Proof. First let $A$ be self-adjoint. We show that every element $C$ in the essential central spectrum of $A$ with respect to $\mathscr{J}$ is in $\mathscr{K}(A)$. There is no loss of generality in assuming for this that $C=0$. Then for every $\varepsilon>0$, there is a projection $E$ in $\mathscr{A}$ such that

$$
\|A E\| \leqq \varepsilon \text { and } E \sim 1
$$

(Example 2.12 and Corollary 3.15).

There are orthogonal projections $E^{\prime}$ and $E^{\prime \prime}$ of sum $E$ such that $E^{\prime} \sim E^{\prime \prime} \sim E$ [9; III, 8, Corollary 2]. By replacing $E$ by $E^{\prime}$, we may assume that $\|A E\| \leqq \varepsilon$ and $E \sim 1-E \sim 1$. Then the element

$$
2^{-1}((E-(1-E)) A(E-(1-E))+A)=E A E+(1-E) A(1-E)
$$

is in $\mathscr{K}^{\prime}(A)$. Now let $E_{1}, \cdots, E_{n}$ be orthogonal projections of sum $E$ with $E_{1} \sim \cdots \sim E_{n} \sim E$, and let $U_{1}, \cdots, U_{n}$ be unitary operators in $\mathscr{A}$ so that the domain support of $(1-E) U_{i}$ equals $E_{i}$. For every unit vector $x$ in the Hilbert space, we have

$$
\begin{aligned}
& \left\|\sum\left\{n^{-1} U_{i}^{-1}(E A E+(1-E) A(1-E)) U_{i} x \mid 1 \leqq i \leqq n\right\}\right\| \\
& \left\|\sum n^{-1} U_{i}^{-1} E A E U_{i} x\right\|+\left\|\sum n^{-1} U_{i}^{-1}(1-E) A(1-E) U_{i} x\right\| \\
& \leqq n\left(n^{-1} \varepsilon\right)+\left\|\sum n^{-1} E_{i} U_{i}^{-1}(1-E) A(1-E) U_{i} E_{i} x\right\| \\
& \leqq \varepsilon+n^{-1}\|A\| .
\end{aligned}
$$

This proves that $\mathscr{K}^{\prime}(A)$ contains an element of norm less than or equal to $\varepsilon+n^{-1}\|A\|$. Because $\varepsilon>0$ and $n$ are arbitrary, the set $\mathscr{K}(A)$ contains 0 . This means that the essential central spectrum of $A$ with respect to $\mathcal{J}$ is contained in $\mathscr{K}(A)$. Hence, the least upper bound $C_{u}$ and the greatest lower bound $C_{l}$ of the essential central spectrum are in $\mathscr{K}(A)$. Since $\mathscr{K}_{\mathcal{F}}(A)$ is the smallest central- 
convex set containing $C_{l}$ and $C_{u}$ (Theorem 4.4) and since $\mathscr{K}(A)$ is central convex, we have that $\mathscr{K}_{\mathcal{J}}(A) \subset \mathscr{K}(A)$.

Now let $A$ be an arbitrary element of $\mathscr{A}$ and let $\phi \in E_{a}(\mathscr{J})$. We may assume that $\phi(A)=0$. We show that 0 is in $\mathscr{K}(A)(\zeta)=$ $\left\{B^{\wedge}(\zeta) \mid B \in \mathscr{C}^{\wedge}(A)\right\}$ for every $\zeta$ in the spectrum of the center. Since $\mathscr{K}(A)(\zeta)$ is compact (Proposition 4.11), there is a $C$ in $\mathscr{K}(A)$ with $\left|C^{\wedge}(\zeta)\right|=\operatorname{glb}\left\{|\alpha| \mid \alpha \in \mathscr{K}^{\wedge}(A)(\zeta)\right\}$. There is no loss of generality in assuming $C^{\wedge}(\zeta) \geqq 0$. We obtain a contradiction by assuming $C^{\wedge}(\zeta)>$ 0 . Indeed, we have that $\phi\left(A+A^{*}\right)=\phi(A)+\phi(A)^{*}=0$. By the preceding paragraph we conclude that $0 \in \mathscr{K}\left(2^{-1}\left(A+A^{*}\right)\right)$ and so there is a sequence $\left\{f_{n}\right\}$ in the subset $\mathscr{E}$ of functions on the unitary operators of $\mathscr{A}$ with $\lim f_{n} \cdot\left(2^{-1}\left(A+A^{*}\right)\right)=0$. We may also assume that $\left\{f_{n} \cdot\left((2 i)^{-1}\left(A-A^{*}\right)\right)\right\}$ converges to a self-adjoint element $B$ in the center [9; III, §5, Problem 2]. Hence, the element $i B$ is in $\mathscr{K}(A)$. However, we must have that $B^{\wedge}(\zeta)=0$. Indeed, if $B^{\wedge}(\zeta) \neq 0$, then the distance to the origin of the line segment $L$ in the complex plane with end-points $C^{\wedge}(\zeta)$ and $i B^{\wedge}(\zeta)$ is less than $C^{\wedge}(\zeta)$. However, this contradicts the definition of $C$ since $L \subset \mathscr{C}(A)(\zeta)$. So we must have that $C^{\wedge}(\zeta)=0$, and hence $0 \in \mathscr{K}^{\wedge}(A)(\zeta)$. The proof is now completed by a compactness argument. Let $\varepsilon>0$ be given. For every $\zeta$ in the spectrum of the center, there a $C_{\zeta}$ in $\mathscr{K}(A)$ and a central projection $P_{\zeta}$ with $P_{\zeta}^{\wedge}(\zeta)=1$ such that $\left\|C_{\zeta} P_{\zeta}\right\| \leqq \varepsilon$. Due to the compactness of the spectrum of the center, we may find $C_{1}, \cdots, C_{n}$ in $\mathscr{K}(A)$ and orthogonal central projections $P_{1}, \cdots, P_{n}$ of sum 1 such that

$$
\left\|\sum C_{i} P_{i}\right\| \leqq \varepsilon
$$

However, $\mathscr{C}(A)$ is central-convex and so $\sum C_{i} P_{i} \in \mathscr{C}(A)$. Since $\varepsilon>0$ is arbitrary and since $\mathscr{K}(\mathscr{A})$ is closed, we have that $0 \in \mathscr{K}(A)$. This completes the first part of the proof.

Conversely, let $C \in \mathscr{K}(A)$. There is no loss of generality in assuming $C=0$. We find $\phi$ in $E_{a}(\mathscr{J})$ with $\phi(A)=0$ : Let $\phi_{0}$ be a state of $\mathscr{A}^{\sim}$ that vanishes on $\mathscr{J}$ (Lemma 4.3). Let $\left\{f_{n}\right\}$ be a sequence of functions in $\mathscr{E}$ such that $\lim f_{n} \cdot A=0$. Let $\phi_{n}$ be the state of $E_{a}(\mathscr{J})$ given by $\phi_{n}(B)=\phi_{0}\left(f_{n} \cdot B\right)$ for every $B$ in $\mathscr{A}$. Due to the compactness of the state space of $\mathscr{A}^{\sim}$ in the $\sigma_{W}\left(\mathscr{A}^{\sim}, \mathscr{A}\right)$ topology, there is a subnet $\left\{\phi_{n_{j}}\right\}$ of $\left\{\phi_{n}\right\}$ and a state $\phi$ of $\mathscr{A}^{\sim}$ such that $\left\{\phi_{n_{j}}(B)\right\}$ converges weakly to $\phi(B)$ for every $B$ in $\mathscr{A}$. Clearly, the state $\phi$ vanishes on $\mathscr{J}$. However, for every $x$ and $y$ in the Hilbert space, we have that

$$
|(\phi(A) x, y)|=\lim _{j}\left|\left(\phi_{n_{j}}(A) x, y\right)\right| \leqq \lim \sup \left\|\phi_{0}\right\|\left\|f_{n_{j}} \cdot A\right\|\|x\|\|y\|=0 \text {. }
$$

This proves that $\phi(A)=0$, and so $0 \in \mathscr{K}_{\mathscr{F}}(A)$. 
algebra and let $A$ be an element of $\mathscr{A}$. Then the convex subset $\mathscr{K}(A)$ of the center is weakly compact.

Proof. For any central ideal $\mathscr{I}$, the set $\mathscr{K}_{\mathcal{F}}(A)$ is weakly compact (Introduction, §4).

Let $A$ be an element in the von Neumann algebra $\mathscr{A}$. Define $\mathscr{C}(A)$ to be the intersection of the weak closure of $\mathscr{K}^{\prime}(A)$ with the center of $\mathscr{A}$. Using the tools we developed here, we can extend the theorem of J. Conway [4] from the case of properly infinite factors to pro perly infinite algebras with arbitrary centers. For this extension the following lemma is needed.

Lemma 4.15. Let $\mathscr{A}$ be a von Neumann algebra on the Hilbert space $H$. Let $f$ be a $\sigma_{w}\left(\mathscr{A}^{\sim}, \mathscr{A}\right)$-continuous hermitian functional on $\mathscr{A}^{\sim}$ (i.e. $f(\phi)$ is real for every $\phi$ in $\mathscr{A}^{\sim}$ which takes hermitian elements of Af into hermitian elements of the center). Then there is an $x \in H$ and a self-adjoint $A \in \mathscr{A}$ such that $f(\phi)=(\phi(A) x, x)$ for every $\phi \in \mathscr{A}^{\sim}$.

Proof. There are $x_{1}, \cdots, x_{n}, y_{1}, \cdots, y_{n}$ in $H$ and $A_{1}, \cdots, A_{n}$ in $\mathscr{A}$ such that $f(\phi)=\sum\left(\phi\left(A_{i}\right) x_{i}, y_{i}\right)$ for all $\phi$ in $\mathscr{A}^{\sim}$ [17; $\S 2$, Introduction]. For each $i$ there are $z_{i j}(1 \leqq j \leqq 4)$ such that

$$
w_{x_{i}, y_{i}}=w_{z_{i 1}}+w_{z_{i 2}}+i\left(w_{z_{i 3}}-w_{z_{i 4}}\right)
$$

where $w_{x_{i}, y_{i}}(B)=\left(B x_{i}, y_{i}\right)$ and $w_{z}=w_{z, z}$ on the center of $\mathscr{A}$ [9; I, 4, Theorem 6 and III, 1, Theorem 4, Corollary]. Then there is an $x$ in $H$ with $w_{x}=\sum_{i, j} w_{z_{i j}}$ [9; III, 1, Theorem 4, Corollary]. For each $i j$, there is a positive element $C_{i j}$ in the center with $\left(B C_{i j} x, x\right)=\left(B z_{i j}, z_{i j}\right)$ for all $B$ in the center (Radon-Nikodym theorem). Thus there is an element $B=\sum A_{i}\left(C_{i 1}-C_{i 2}+i\left(C_{i 3}-C_{i 4}\right)\right)$ in $\mathscr{A}$ with $f(\phi)=(\phi(B) x, x)$ for every $\phi$ in $\mathscr{A}^{\sim}$. If $\phi^{*}(B)=\phi\left(B^{*}\right)^{*}$ for $\phi \in \mathscr{A}^{\sim}$, then

$$
\left(\dot{\phi}\left(B^{*}\right)^{*} x, x\right)=f\left(\dot{\phi}^{*}\right)=f(\dot{\phi})^{-}=(\dot{\phi}(B) x, x)^{-}
$$

for every $\phi$ in $\mathscr{A}^{\sim}$ implies that $f(\phi)=\left(\dot{\phi}\left(B^{*}\right) x, x\right)=(\phi(B) x, x)$ for every $\phi$ in $\mathscr{A}$. Hence, $f(\phi)=(\phi(A) x, x)$ for every $\phi$ in $\mathscr{A}^{\sim}$. Here $A=$ $2^{-1}\left(B+B^{*}\right)$.

THEOREM 4.16. Let $\mathscr{A}$ be a properly infinite von Neumann algebra, and let $\mathscr{F}$ be the ideal of finite elements of $\mathscr{A}$; then $\mathscr{C}(A)=$ $\mathscr{K}_{\mathcal{S}}(A)$ for every $A$ in $\mathscr{A}$.

Proof. One may prove the theorem using the same steps (with 
appropriate modifications) that Conway [4] employed in his proof for factor algebras. We content ourselves with pointing out the appropriate steps. Let $\mathscr{C}$ be the set of all states of $\mathscr{A}^{\sim}$ such that $\phi(A) \in$ $\mathscr{C}(A)$ for all $A$ in $\mathscr{A}$. For every $A \in \mathscr{A}$ and $A_{0} \in \mathscr{C}(A)$, there is a $\phi \in \mathscr{C}$ such that $\phi(A)=A_{0}$. This uses the $\sigma_{w}\left(\mathscr{A}^{\sim}, \mathscr{A}\right)$-topology instead of the weak *-topology of the dual of $\mathscr{A}$ [4; Lemma 5]. The set $\mathscr{C}(A)$ is equal to $\{0\}$ for every $A \in \mathscr{J}$ [4; Lemma 6]. Hence, the set $\mathscr{C}$ is a subset of $E_{a}(\mathscr{F})$. But if $A$ is self-adjoint and $\phi \in$ $E_{a}(\mathscr{F})$, then $\phi(A) \in \mathscr{C}(A)$ since the least upper bound and the greatest lower bound of the essential central spectrum of $A$ with respect to $\mathscr{J}$ are in $\mathscr{C}(A)$ (argue as in [4; Lemma 4] based on Proposition 3.13) and since $\mathscr{C}(A)$ is central-convex (use the fact that $\mathscr{K}^{\prime}(A)$ is centralconvex). If there is $\phi_{0}$ in $E_{a}(\mathscr{J})$ but not in the $\sigma_{W}\left(\mathscr{A}^{\sim}, \mathscr{A}\right)$-compact convex set $\mathscr{C}$, then there is a $\sigma_{W}\left(\mathscr{A}^{\sim}, \mathscr{A}\right)$-continuous hermitian functional on $\mathscr{A}^{\sim}$ which strongly separates $\phi_{0}$ from $\mathscr{C}$. However, every $\sigma_{W}\left(\mathscr{A}^{\sim}, \mathscr{A}\right)$-continuous hermitian functional $f$ of $\mathscr{A}^{\sim}$ is of the form $f(\phi)=(\phi(A) x, x)$ for some fixed self-adjoint $A$ in $\mathscr{A}$ and some vector $x$ in the Hilbert space. This contradicts the fact that $\dot{\phi}_{0}(A) \in \mathscr{C}(A)$ and so that $\dot{\phi}_{0}(A)=\phi(A)$ for some $\phi \in \mathscr{C}$. Hence, $\mathscr{C}=E_{a}(\mathscr{I})$ and $\mathscr{K}_{\mathcal{F}}(A)=\mathscr{C}(A)$.

CoRollary 4.17. Let $\mathscr{A}$ be a $\sigma$-finite properly infinite von Neumann algebra; then $\mathscr{K}(A)=\mathscr{C}(A)$ for every $A$ in $\mathscr{A}$.

Proof. The ideal generated by the finite elements of $\mathscr{A}$ is the strong radical of $\mathscr{A}$. The corollary then follows from Theorems 4.12 and 4.16.

5. Applications. Using the notions of essential central spectrum and essential numerical range, we can extend some theorems on commutators and derivations to arbitrary properly infinite von Neumann algebras. These theorems are known for the algebra of all bounded linear operators on a Hilbert space, which is generally assumed to be separable, but the techniques employed there also suffice here.

A linear map $\delta$ of an algebra is said to be a derivation if $\delta(A B)=$ $A \delta(B)+\delta(A) B$ for every $A$ and $B$ in the algebra. S. Sakai [27] proved that every derivation $\delta$ of a von Neumann algebra $\mathscr{A}$ is inner in the sense that there is an $A$ in $\mathscr{A}$ such that $\delta(B)=A B-B A$ for every $B$ in $\mathscr{A}$. The next proposition is due to J. G. Stampfli [29] for the algebra of bounded linear operators on a Hilbert space. His technique suffices here. 
Neumann algebra is not uniformly dense in the algebra.

Proof. Since every von Neumann algebra may be written as a product of a finite and a properly infinite von Neumann algebra, it is sufficient to consider these two cases separately. If the algebra is finite, then the range of the derivation is contained in the set of elements whose canonical operator-valued trace vanishes. So the range of a derivation cannot be dense. If the von Neumann algebra $\mathscr{A}$ is properly infinite and the derivation $\delta$ on $\mathscr{A}$ is given by $\delta(B)=$ $A B-B A$, then we construct an operator that is not in the closure of the range of $\delta$. Let $A_{0}$ be a central element such that $\left(A-A_{0}\right)(\mathscr{J}(\zeta))$ is neither left nor right invertible for all $\zeta$ in the spectrum of the center. Here $\mathscr{J}$ is the strong radical of $\mathscr{A}$ (Theorem 3.5). Because $\delta(B)=\left(A-A_{0}\right) B-B\left(A-A_{0}\right)$ for all $B \in \mathscr{A}$, we may assume $A_{0}=0$. There are sequences $\left\{E_{n}\right\}$ and $\left\{F_{n}\right\}$ of mutually orthogonal projections in $\mathscr{A}$ such that $E_{n} \sim 1 \sim F_{n},\left\|A E_{n}\right\| \leqq n^{-1}$, and $\left\|F_{n} A\right\| \leqq n^{-1}$ for for every $n=1,2, \cdots$ (Example 2.12 and Corollary 3.16). Then there is a partial isometry $U$ in $\mathscr{A}$ with domain support $E=\sum E_{i}$ and range support $F=\sum F_{i}$ such that $U E_{i}=F_{i} U$. We show that $\alpha=$ $\|U-\delta(B)\| \geqq 1$ for every $B \in \mathscr{A}$. Indeed, for every $n=1,2, \cdots$, we have that

$$
1=\left\|F_{n} U E_{n}\right\| \leqq\left\|F_{n}(U-\delta(B)) E_{n}\right\|+\left\|F_{n} \delta(B) E_{n}\right\| \leqq \alpha+2 n^{-1}\|B\| \cdot
$$

Hence the open ball of radius 1 about $U$ does not meet the range of $\delta$.

In [18], we showed that an element $A$ in a properly infinite von Neumann algebra $\mathscr{A}$ is a commutator in $\mathscr{A}$ (i.e. there are elements $B$ and $C$ with $A=B C-C B$ ) provided $0 \in \mathscr{K}(A)$. We can also prove that $0 \in \mathscr{K}(A)$ provided $A=B C-C B$ and $\pm\left(B^{*} B-B B^{*}\right)$ is a positive operator in $\mathscr{A}$. Now an element $A$ is said to be a self-adjoint commutator if $A=B C-C B$ with $B=B^{*}$. H. Radjavi [25] characterized those self-adjoint elements in the algebra $B(H)$ of all bounded linear operators on a separable Hilbert space $H$ which are self-adjoint commutators and J. Anderson [1] recently announced that he has completely characterized self-adjoint commutators in $B(H)$. We prove a proposition in this direction for properly infinite von Neumann algebras using a matrix calculation of M. David [5].

Proposition 5.2. Let $\mathscr{A}$ be a properly infinite von Neumann algebra and let $A$ be a self-adjoint element in $\mathscr{A}$. If 0 is in the essential central spectrum of $A$ with respect to the strong radical of $\mathscr{A}$, then $A$ is a self-adjoint commutator in $\mathscr{A}$. 
Proof. There is a sequence $\left\{E_{n}\right\}$ of orthogonal projections with $E_{n} \sim 1$ and $\left\|A E_{n}\right\| \leqq 1 / n$ ! for all $n=1,2, \cdots$ (Lemma 3.16 and Example 2.12). Thus, $\left\|E_{m} A E_{n}\right\| \leqq \min \{1 / m !, 1 / n !\}$. Then the matrix calculation of M. David [5; Theorem 3] is applicable.

Acknowledgement. The author would like to thank Professor Carl Pearcy for bringing J. G. Stampfli's result [29] to his attention and for suggesting extending it to von Neumann algebras. He also wishes to thank Professor Stampfli for a letter in which he outlined his proof (cf. Introduction $\$ 5$ ).

Added in Proof, August 24, 1972. We have obtained a better version of Proposition 5.2 by showing that $A$ is a self-adjoint commutator whenever 0 is in the essential central range of $A$.

\section{REFERENCES}

1. J. Anderson, Commutators and the essential numerical range, Notices Amer. Math. Soc., 18 (1971), no. 6 82-47-14.

2. S. Berberian and G. H. Orland, On the closure of the numerical range of an operator, Proc. Amer. Math. Soc., 18 (1967), 499-503.

3. A. Brown, C. Pearcy and D. Topping, Commutators and the strong radical, Duke Math. J., 53 (1968), 853-859.

4. J. Conway, The numerical range and a certain convex set in an infinite factor, J. Functional Analysis, 5 (1970), 428-435.

5. M. David, On a certain type of commutator, J. Math. Mech., 19 (1969/70), 665-680.

6. D. Deckard and C. Pearcy, Continuous matrix-valued functions on a Stonian space, Pacific J. Math., 14 (1964), 857-869.

7. J. Dixmier, Sur certains espaces considerés par M. H. Stone Summa Brasil. Math. 2 fasc., 11 (1951), 151-182.

8. ——, Applications घ dans les anneaux d'opérateurs, Compos. Math., 10 (1952), $1-55$.

9. —_Les algèbres d'opérateurs dans l'espace hilbertien, Cahiers Scientifiques, fasc. 25 Gauthier-Villars, Paris, 1969.

10. L Les $C^{*}$-algèbres et leurs réprésentations , Cahier Scientifiques, fasc. 29, Gauthier-Villars, Paris, 1964.

11. P. Fillmore and J. G. Stampfli, J. P. Williams, On the essential numerical range, the essential spectrum, and a problem of Halmos.

12. J. Glimm, A Stone-Weierstrass theorem for $C^{*}$-algebras, Ann. of Math., (2) 72 (1960), Ann. of Math., (2) $\mathbf{7 2}$ (1960), 216-244.

13. M. Goldman, Structure of $A W^{*}$-algebras of type I, Duke J. Math., 23 (1956), 2334 .

14. H. Halpern, A spectral decomposition for self-adjoint elements in the maximum GCR ideal of a von Neumann algebra with applications to non-commutative integration theory, Trans. Amer. Math. Soc., 133 (1968), 281-306.

15. Commutators in a properly infinite von Neumann algebra, Trans. Amer. Trans. Amer. Math. Soc., 139 (1969), 55-73.

16. - Module homomorphisms of a von Neumann algebra into its center, Trans. Amer. Math. Soc., 140 (1970), 183-193.

17. Irreducible module homomorphisms of a von Neumann algebra into its 
center, Trans. Amer. Math. Soc., 140 (1969), 194-221.

18. , Commutators modulo the center in a properly infinite von Neumann algebra, Trans. Amer. Math. Soc., 150 (1970), 55-68.

19. - A generalized dual for a $C^{*}$-algebra, Trans. Amer. Math. Soc., 153 (1971), $139-156$.

20. R. V. Kadison, Derivations of operator algebras, Ann. of Math., 83 (1966), 280-293. 21. I. Kaplansky, Projections in Banach algebras, Ann. of Math., (2) 53 (1951), 235249.

22. - Algebras of type I, Ann. of Math. (2), 56 (1952), 460-472.

23. — Modules over operator algebras, Amer. J. Math., 75 (1953), 839-858.

24. Y. Misonou, On a weakly central operator algebra, Tohôku Math. J., (2) 4 (1952), 194-202.

25. H. Radjavi, Structure of $A^{*} A-A A^{*}$, J. Math. Mech., 16 (1966), 19-26.

26. C. Rickart, General theory of Banach algebras, The University Series in Higher Math., Van Nostrand, Princeton, N. J., 1960.

27. S. Sakai, Derivations of $W^{*}$-algebras, Ann. of Math., 83 (1966), 273-279.

28. I. E. Segal, A non-commutative extension of abstract integration, Ann. of Math., (2) 57 (1953), 401-457.

29. J. G. Stampfii, The range of a derivation (to appear).

30. J. Tomiyama, Generalized dimension function for $W^{*}$-algebras of infinite type, Tohôku Math. J., 10 (1958), 121-129.

31. W. Wils, Two-sided ideals in $W^{*}$-algebras, J. Reine Agnew. Math., 244 (1971), $55-68$.

32. F. B. Wright, A reduction for algebras of finite type, Ann. of Math., 60 (1954), $560-570$.

Received April 15, 1971. This research was partially supported by the National Science Foundation.

UnIVERSity of Cincinnati 


\section{PACIFIC JOURNAL OF MATHEMATICS}

\section{EDITORS}

\author{
H. SAMELSON \\ Stanford University \\ Stanford, California 94305 \\ C. R. HOBBY \\ University of Washington \\ Seattle, Washington 98105
}

\author{
J. DugundJI \\ Department of Mathematics \\ University of Southern California \\ Los Angeles, California 90007
}

\author{
RICHARD ARENS \\ University of California \\ Los Angeles, California 90024
}

\section{ASSOCIATE EDITORS}
E. F. BECKENBACH
B. H. NeumanN
F. WOLF
K. YoSHIDA

\section{SUPPORTING INSTITUTIONS}

\author{
UNIVERSITY OF BRITISH COLUMBIA \\ CALIFORNIA INSTITUTE OF TECHNOLOGY \\ UNIVERSITY OF CALIFORNIA \\ MONTANA STATE UNIVERSITY \\ UNIVERSITY OF NEVADA \\ NEW MEXICO STATE UNIVERSITY \\ OREGON STATE UNIVERSITY \\ UNIVERSITY OF OREGON \\ OSAKA UNIVERSITY
}

\author{
UNIVERSITY OF SOUTHERN CALIFORNIA \\ STANFORD UNIVERSITY \\ UNIVERSITY OF TOKYO \\ UNIVERSITY OF UTAH \\ WASHINGTON STATE UNIVERSITY \\ UNIVERSITY OF WASHINGTON \\ $* * * *$
$*$
AMERICAN MATHEMATICAL SOCIETY
NAVAL WEAPONS CENTER
}

The Supporting Institutions listed above contribute to the cost of publication of this Journal, but they are not owners or publishers and have no responsibility for its content or policies.

Mathematical papers intended for publication in the Pacific Journal of Mathematics should be in typed form or offset-reproduced, (not dittoed), double spaced with large margins. Underline Greek letters in red, German in green, and script in blue. The first paragraph or two must be capable of being used separately as a synopsis of the entire paper. The editorial "we" must not be used in the synopsis, and items of the bibliography should not be cited there unless absolutely necessary, in which case they must be identified by author and Journal, rather than by item number. Manuscripts, in duplicate if possible, may be sent to any one of the four editors. Please classify according to the scheme of Math. Rev. Index to Vol. 39. All other communications to the editors should be addressed to the managing editor, Richard Arens, University of California, Los Angeles, California, 90024.

50 reprints are provided free for each article; additional copies may be obtained at cost in multiples of 50 .

The Pacific Journal of Mathematics is published monthly. Effective with Volume 16 the price per volume (3 numbers) is $\$ 8.00$; single issues, $\$ 3.00$. Special price for current issues to individual faculty members of supporting institutions and to individual members of the American Mathematical Society: $\$ 4.00$ per volume; single issues $\$ 1.50$. Back numbers are available.

Subscriptions, orders for back numbers, and changes of address should be sent to Pacific Journal of Mathematics, 103 Highland Boulevard, Berkeley, California, 94708.

PUBLISHED BY PACIFIC JOURNAL OF MATHEMATICS, A NON-PROFIT CORPORATION

Printed at Kokusai Bunken Insatsusha (International Academic Printing Co., Ltd.), 270, 3-chome Totsuka-cho, Shinjuku-ku, Tokyo 160, Japan. 


\section{Pacific Journal of Mathematics}

\section{Vol. 43, No. 2 \\ April, 1972}

Arne P. Baartz and Gary Glenn Miller, Souslin's conjecture as a problem on the real line....................................... 277

Joseph Barback, On solutions in the regressive isols ............... 283

Barry H. Dayton, Homotopy and algebraic K-theory ................ 297

William Richard Derrick, Weighted convergence in length ............ 307

M. V. Deshpande and N. E. Joshi, Collectively compact and semi-compact sets of linear operators in topological vector spaces ............. 317

Samuel Ebenstein, Some $H^{p}$ spaces which are uncomplemented in $L^{p} \ldots . .327$

David Fremlin, On the completion of locally solid vector lattices ......... 341

Herbert Paul Halpern, Essential central spectrum and range for elements of

a von Neumann algebra............................... 349

G. D. Johnson, Superadditivity intervals and Boas' test ............. 381

Norman Lloyd Johnson, Derivation in infinite planes . . . . . . . . . . . 387

V. M. Klassen, The disappearing closed set property .............. 403

B. Kuttner and B. N. Sahney, On the absolute matrix summability of Fourier series ........................................... 407

George Maxwell, Algebras of normal matrices................... 421

Kelly Denis McKennon, Multipliers of type $(p, p) \ldots \ldots \ldots \ldots \ldots \ldots . . \ldots 29$

James Miller, Sequences of quasi-subordinate functions ............. 437

Leonhard Miller, The Hasse-Witt-matrix of special projective varieties ..... 443

Michael Cannon Mooney, A theorem on bounded analytic functions ...... 457

M. Ann Piech, Differential equations on abstract Wiener space .......... 465

Robert Piziak, Sesquilinear forms in infinite dimensions ............. 475

Muril Lynn Robertson, The equation $y^{\prime}(t)=F(t, y(g(t))) \ldots \ldots \ldots \ldots .483$

Leland Edward Rogers, Continua in which only semi-aposyndetic

subcontinua separate ............................... 493

Linda Preiss Rothschild, Bi-invariant pseudo-local operators on Lie

groups ...................................... 503

Raymond Earl Smithson and L. E. Ward, The fixed point property for

arcwise connected spaces: a correction ...................... 511

Linda Ruth Sons, Zeros of sums of series with Hadamard gaps .......... 515

Arne Stray, Interpolation sets for uniform algebras............... 525

Alessandro Figà-Talamanca and John Frederick Price, Applications of random Fourier series over compact groups to Fourier multipliers .. 